ARTICLE

Received 13 Dec 2013 | Accepted 1 Apr 2014 | Published 7 May $2014 \quad$ DOl: 10.1038/ncomms4780

\title{
Single-vesicle architecture of synaptobrevin2 in astrocytes
}

Priyanka Singh ${ }^{1}$, Jernej Jorgačevski ${ }^{1,2}$, Marko Kreft ${ }^{1,2,3}$, Vladimir Grubišić ${ }^{4}$, Randy F. Stout Jr ${ }^{4,5}$, Maja Potokar ${ }^{1,2}$, Vladimir Parpura ${ }^{4,6} \&$ Robert Zorec ${ }^{1,2}$

Exocytic transmitter release is regulated by the SNARE complex, which contains a vesicular protein, synaptobrevin2 (Sb2). However, Sb2 vesicular arrangement is unclear. Here we use super-resolution fluorescence microscopy to study the prevalence and distribution of endogenous and exogenous Sb2 in single vesicles of astrocytes, the most abundant glial cells in the brain. We tag Sb2 protein at $\mathrm{C}$ - and $\mathrm{N}$ termini with a pair of fluorophores, which allows us to determine the Sb2 length and geometry. To estimate total number of Sb2 proteins per vesicle and the quantity necessary for the formation of fusion pores, we treat cells with ATP to stimulate $\mathrm{Ca}^{2+}$-dependent exocytosis, increase intracellular alkalinity to enhance the fluorescence presentation of yellow-shifted pHluorin $(\mathrm{YpH})$, appended to the vesicle lumen domain of $\mathrm{Sb2}$, and perform photobleaching of $\mathrm{YpH}$ fluorophores. Fluorescence intensity analysis reveals that the total number of endogenous Sb2 units or molecules per vesicle is $\leq 25$.

\footnotetext{
${ }^{1}$ Laboratory of Neuroendocrinology-Molecular Cell Physiology, Institute of Pathophysiology, Faculty of Medicine, University of Ljubljana, 1000 Ljubljana, Slovenia. ${ }^{2}$ Celica Biomedical Center, 1000 Ljubljana, Slovenia. ${ }^{3}$ Department of Biology, Biotechnical Faculty, University of Ljubljana, 1000 Ljubljana, Slovenia. ${ }^{4}$ Department of Neurobiology, Center for Glial Biology in Medicine, University of Alabama, Birmingham, Alabama 35294, USA. ${ }^{5}$ The Dominick P. Purpura Department of Neuroscience, Albert Einstein College of Medicine, Bronx, New York 10461, USA. ${ }^{6}$ Department of Biotechnology, University of Rijeka, 51000 Rijeka, Croatia. Correspondence and requests for materials should be addressed to V.P. (email: vlad@uab.edu) or to R.Z. (email: robert.zorec@mf.uni-lj.si).
} 
strocytes provide metabolic support to neurons, actively tune synaptic activity and influence the microcirculation of the brain ${ }^{1}$. A key process mediating astrocyte communication with neighbouring cells is regulated exocytosis. This multi-stage, spatially and temporally highly organized process consists of many steps leading to merger between secretory organelles and the plasma membrane. Astrocytic secretory organelles may contain gliotransmitters (peptides, amino acids and/or nucleotides), membrane transporters, channels and/or other signalling molecules ${ }^{2,3}$. Distinct secretory organelle types $^{4-6}$ can be engaged in regulated exocytosis ${ }^{7}$ and they are differentially regulated. Vesicular exocytosis is mediated by $\mathrm{Sb} 2$ (ref. 8), also referred to as VAMP2, while lysosomal exocytosis is thought to be governed by VAMP7 (ref. 9). Thus, to understand the nature of the exocytotic process and its role in astrocyte communication, one needs to understand secretory organelle architecture at the highest possible spatial resolution in living cells. Individual vesicles can be studied by labelling proteins that allow the vesicular fusion process. Therefore, one can mark $\mathrm{Sb} 2$, which is typically present on the vesicle membrane in astrocytes ${ }^{10,11}$.

Our knowledge regarding the architecture of biological organelles and their function at the cellular and subcellular level in living cells arises mainly from studies using microscopic techniques, such as confocal laser scanning microscopy $(\mathrm{CLSM})^{12,13}$. However, the resolution limit of CLSM, dictated by diffraction ${ }^{14}$, impedes the ability to study single-cell organelles smaller than the limit of transverse and axial resolution ( $\sim 200 \mathrm{~nm}$ and $\sim 500 \mathrm{~nm}$, respectively). This limit can be overcome by super-resolution microscope techniques ${ }^{15}$, which include structured illumination microscopy (SIM) with improved transverse resolution to $\sim 100 \mathrm{~nm}^{16}$, thus allowing punctiform object measurements at the nanoscale level. Furthermore, as exploited in the present work, SIM can also report on interfluorophore distances at a fraction of its resolution limit.

To study how Sb2 is structurally integrated and distributed in single vesicles, we examined the arrangement of this vesicle membrane protein in fixed and living cultured astrocytes. As a dimensional calibration, we measured the length between two fluorescent tags placed at two ends of a Sb2 protein at the singlevesicle level. Analysis of the intensity of fluorescent tags, reporting on the number of molecules, revealed a multimodal distribution with equally interspaced peaks. We conclude that there are $\leq 25$ molecules of endogenous Sb2 per vesicle in astrocytes.

\section{Results}

Arrangement of $\mathrm{Sb} 2$ on a single vesicle. To describe the structural integration of $\mathrm{Sb} 2$ proteins in a single vesicle at the nanometer scale, we designed a genetically modified protein chimera, yellow synaptopHluorin (YSpH) with yellow-shifted superecliptic pHluorin (YpH; a derivative of pHluorin $^{17}$, a modified enhanced green fluorescent protein (EGFP)) targeted to the vesicle lumen (labelled as A in Fig. 1a), by attaching it to the $\mathrm{C}$ terminus of Sb2 via an 8-amino-acid (aa) linker (Supplementary Fig. 1a). The $\mathrm{N}$-terminus of the same protein was marked with an anti-Sb2 antibody (recognizing aa 1-18 of rat Sb2), which was tagged with secondary antibodies conjugated with Atto 594, a rhodamine derivative dye, that has excitation and emission wavelengths spectrally compatible with $\mathrm{YpH}$ (labelled as B in Fig. 1a). By considering the length of the $\mathrm{Sb} 2$ protein to be $46 \mathrm{~nm}$ in its

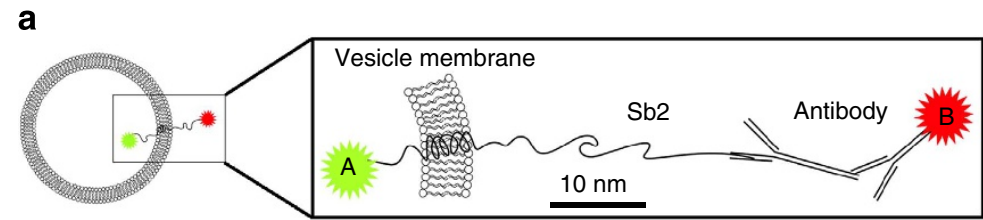

b
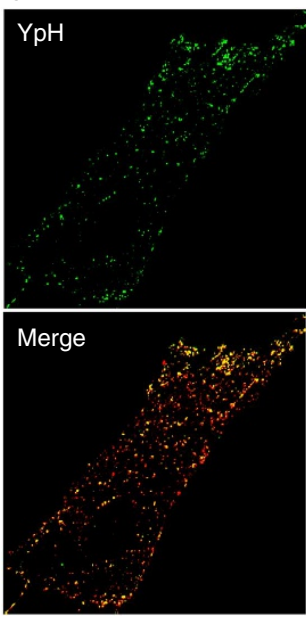
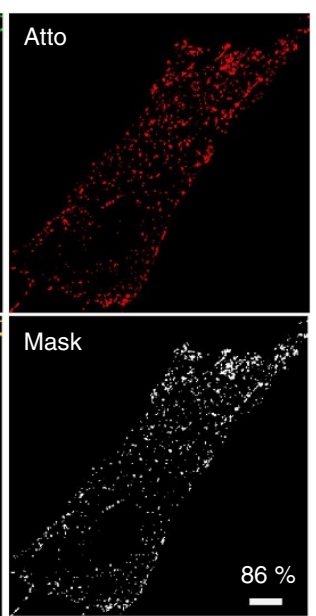

C
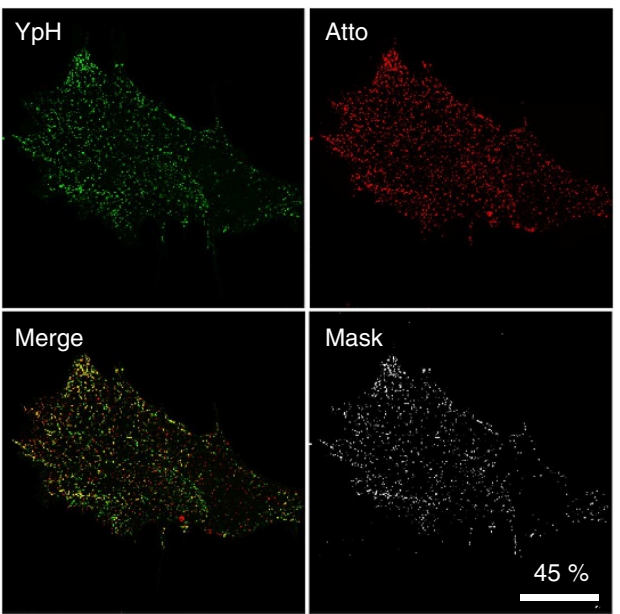

Figure 1 | Vesicle-associated membrane protein Sb2 fluorescently labelled at the luminal and cytoplasmic sides of the vesicle membrane. (a) The diagram represents an astrocytic vesicle (left) in which a version of Sb2 is expressed, with a fluorescent protein in the vesicle lumen (fluorophore A, that is, $\mathrm{YpH}$ ) and with an immunolabelled cytoplasmic domain (fluorophore B, that is, Atto 594). The boxed diagram on right shows the architecture of Sb2 across the vesicle membrane, drawn to scale by considering the length of Sb2 ${ }^{18,19}$. b and $\mathbf{c}$ are images from CLSM and SIM, respectively. To stain $\mathrm{Sb} 2$ in vesicle lumen, astrocytes were transfected with a plasmid encoding YSpH (green). To stain the N-terminal part of Sb2 located on the cytosolic side of the vesicle membrane, primary antibody against Sb2 and a secondary antibody conjugated to Atto 594 (Atto; red) were used. Merge panels show overlay of $\mathrm{YSpH}$ and Atto signals arising from the vesicle lumen and the cytoplasm. Mask panels show only the colocalized pixels between YpH and Atto. Bar, $10 \mu \mathrm{m}$. Note the smaller amount of colocalization (percent, shown for representative single astrocytes) in the SIM image than in the CLSM image. 
elongated or uncoiled form ${ }^{18-21}$ and the average length of an IgG antibody molecule (between $11.5 \mathrm{~nm}$ and $14 \mathrm{~nm}$ for two different configurations) $^{22}$, we predicted a structural arrangement as shown in Fig. 1a. As controls, we made additional genetically encoded Sb2 constructs. To avoid the use of antibodies, we fused $\mathrm{YSpH}$ with red fluorescent protein (mCherry) on the cytoplasmic vesicle side (that is, N-terminus) of $\mathrm{Sb} 2$ (mCherry-YSpH) (Supplementary Fig. 2). In this construct, inter-fluorophore (centre-centre) distance of $\sim 54 \mathrm{~nm}$ is predicted, where one considers an average aa residue size of $0.4 \mathrm{~nm}$ (ref. 20) (thus, for the full length of 116 aa of Sb2 along with 8 aa of the linker, there is total of 124 aa $\times 0.4 \mathrm{~nm}=49.6 \mathrm{~nm}$ ) and EGFP size of $4.7 \mathrm{~nm}^{23}$ along with an assumption of a similar size for mCherry. It should be noted that for $\mathrm{YSpH}$ construct decorated with the primary antibody against $\mathrm{Sb} 2$ and Atto-labelled secondary antibody, the $\mathrm{YpH}$-Atto inter-fluorophore distance is predicted to be $\sim 75$ $80 \mathrm{~nm}$ (assuming a linear arrangement of two antibody molecules in shorter and longer configurations, respectively, as well as a negligible size of Atto in comparison with EGFP). We also prepared another chimeric protein (YSpH-7aa-mCherry) (Supplementary Fig. 2) that had mCherry attached, via a 7-aa residue linker $(\sim 2.8 \mathrm{~nm})$, to $\mathrm{C}$ terminus of $\mathrm{YpH}$, with a predicted inter-fluorophore distance up to $\sim 7.5 \mathrm{~nm}$.

We transfected astrocytes with a plasmid encoding $\mathrm{YSpH}$ (shown in green on images, $\mathrm{YpH}$ ) and subsequently exposed these cells to immunocytochemical labelling with primary antibodies against $\mathrm{Sb} 2$ followed by fluorescently tagged secondary antibodies (Atto 594; shown in red in Fig. 1a). Antibody binds to exogenously expressed $\mathrm{Sb} 2$, that is, $\mathrm{YpH}$, as well as its endogenous nonfluorescent form. As shown in Fig. 1, we determined the degree of colocalization (\%) between $\mathrm{YpH}$ and antibodyconjugated Atto, which significantly differed $(P<0.001$; Student's $t$-test) when obtained by CLSM $(83 \pm 2 \%, n=20$ cells $)$ and SIM ( $40 \pm 4 \%, n=9$ cells). The reduced colocalization in SIM images is consistent with the idea that $\mathrm{YpH}$ and Atto fluorophores, associated with imaged structures or vesicles, are present in close proximity, not overlapping completely ${ }^{24}$. To verify this notion, we further studied individual fluorescent puncta, representing single vesicles. Chemical fixation (see Methods) of the cells enhanced $\mathrm{YpH}$ signal due to $\mathrm{pH}$ equilibration between vesicles and the extracellular medium.

Pairs of $\mathrm{YpH}$ and Atto SIM signals exhibit different patterns (Fig. 2a-d). After eliminating $\mathrm{YpH}$ and Atto signals that were $>200 \mathrm{~nm}$ apart from each other (15-20\% of the puncta), four pattern categories, in descending preponderance, were observed: (a) partial overlapping $\mathrm{YpH}$ and Atto signals (Fig. 2a); (b) partial overlapping $\mathrm{YpH}$ and Atto signals, as in pattern (a), but the average Atto signal exhibited higher intensity than the $\mathrm{YpH}$ signal (Fig. 2b); (c) overlapping $\mathrm{YpH}$ and Atto signals (Fig. 2c); and (d) Atto enveloped $\mathrm{YpH}$ signal, which in cross-sectional fluorescence profiles may appear as a $\mathrm{YpH}$ signal sandwiched between two Atto signals (Fig. 2d). The proportions of these patterns were as follows: for (a) $44 \pm 2 \%$; (b) $34 \pm 2 \%$; (c) $15 \pm 3 \%$; and (d) $7 \pm 1 \%$ ( $n=349$ vesicles, 3 cells) (Fig. 2 e). All arrangements were further studied by plotting normalized intensity line profiles for each of these arrangements (Fig. 2, mid column), which were used to measure the distance between peaks of Gaussian curves, fitted to the intensity of $\mathrm{YpH}$ and Atto signals. The frequency distribution plot for the distance, measured in each pair of $\mathrm{YpH}$ and Atto fluorophores, yielded a mean value of $65 \pm 2 \mathrm{~nm}$ ( $n=541,5$ cells) (Fig. 2f). The measured distance, when compared with predicted values $(75-80 \mathrm{~nm})$, could represent an underestimate if the orientation of Sb2 and associated antibodies are not parallel to the focal plane and/or if their configurations are not so 'linear' as indicated in Fig. 1a (that is, antibodies assume angles and Sb2 coils).
To further verify that this measurement of inter-fluorophore distance is associated with the distance between $\mathrm{C}$ - and $\mathrm{N}$ termini of $\mathrm{Sb} 2$ across the vesicle membrane, we transfected astrocytes to express mCherry- $\mathrm{YSpH}$, an additional $\mathrm{Sb} 2$ construct (Supplementary Fig. 2), having red fluorescent protein (mCherry) appended to the $\mathrm{N}$ terminus (located at the vesicular cytosolic side) of $\mathrm{YSpH}$; $\mathrm{YpH}$ at the $\mathrm{C}$ terminus is located in the vesicular lumen. The results revealed a mean inter-fluorophore (mCherry$\mathrm{YpH})$ distance of $57 \pm 1 \mathrm{~nm}(n=403$ vesicles, 7 cells; Fig. $3 \mathrm{~d})$, which is in good agreement with the predicted inter-fluorophore distance $(54 \mathrm{~nm})$ in this Sb2 chimera, but significantly different $(P=0.004$; analysis of variance (ANOVA) from YpH-Atto measurements in Fig. 2f. This difference can likely be attributed to the length of attached antibodies when studying $\mathrm{YpH}$-Atto pairs. It cannot be ruled out that the mCherry-YpH measurements may represent the distance between fluorophores of two different Sb2 molecules located on adjacent vesicles, although this scenario is unlikely. As an additional cross-check measure, we expressed a chimeric protein with mCherry appended via a 7-aa linker to $\mathrm{C}$ terminus of $\mathrm{YpH}$ (YSpH-7aa-mCherry) (Supplementary Fig. 2) and recorded an inter-fluorophore distance of $0 \pm 5 \mathrm{~nm}$ ( $n=338$ vesicles, 6 cells; Fig. $3 \mathrm{~h})$, a measurement that is consistent with the predicted value $(7.5 \mathrm{~nm})$.

Diameter measurements of pHluorin-laden vesicles. We next assessed vesicular diameter using an approach involving filling the vesicle lumen in live astrocytes using LysoTracker Red DND99 , which stains acidic organelles within a cell ${ }^{25}$. The specificity of monitoring secretory vesicles was obtained by transfecting astrocytes to express $\mathrm{YSpH}$ and hence label $\mathrm{Sb} 2$-positive vesicles, before their exposure to LysoTracker. Subsequently, we analysed only dually, YSpH and LysoTracker, labelled puncta or vesicles (Fig. 4a,b) by obtaining Gaussian curves of the intensity profiles along a line section through the equatorial plane of such vesicles (Fig. 4c). The YSpH Gaussian curves of individual puncta were narrower and eccentrically positioned when compared with those of matching individual LysoTracker puncta, indicating that $\mathrm{YSpH}$ is non-uniformly distributed and does not completely disclose vesicular lumen (Fig. 4c). Nonetheless, by measuring the full width at half maximum intensity (FWHM) of the Gaussian curve of red LysoTracker fluorescence (Fig. 4c,d), we determined the apparent diameter of astrocytic vesicles of $305 \pm 3 \mathrm{~nm}$ ( $n=129$ vesicles, 9 cells). It should be noted that this estimate of the vesicle diameter, which is in reasonable agreement with the previously obtained measurements or Sb2-laden vesicles in live astrocytes $^{26}$, is based on only a fraction of LysoTracker-positive vesicles in which $\mathrm{YpH}$ signal is significant, due to either a relatively high copy number of $\mathrm{YSpH}$ molecules and/or a relatively more alkaline vesicle lumen. In addition, LysoTrackerbased measurement may overestimate vesicular diameter, as is the case when measuring the diameter of standard fluorescent beads (nominally $200 \mathrm{~nm}$ in diameter with measured FWHM of $\sim 255 \mathrm{~nm}$, Supplementary Fig. 3c). Nonetheless, the Sb2-laden vesicles that we have interrogated in live astrocytes have an apparent size of $\sim 300 \mathrm{~nm}$ in diameter.

The number of exogenous $\mathrm{Sb} 2$ molecules on single vesicles. To learn about the putative number of Sb2 molecules per vesicle or fluorescent punctum, we initially made a comparative analysis of the intensities of fluorophores that were used to label Sb2 and of those obtained from standard, dye-loaded spherical latex particles, that is, green fluorescent beads (Supplementary Fig. 3). Close inspection of the frequency histograms of fluorescence intensities of beads (Supplementary Fig. 3d) and fluorophore-tagged Sb2 (YpH, Supplementary Fig. 3e; Atto, Supplementary Fig. 3f) 

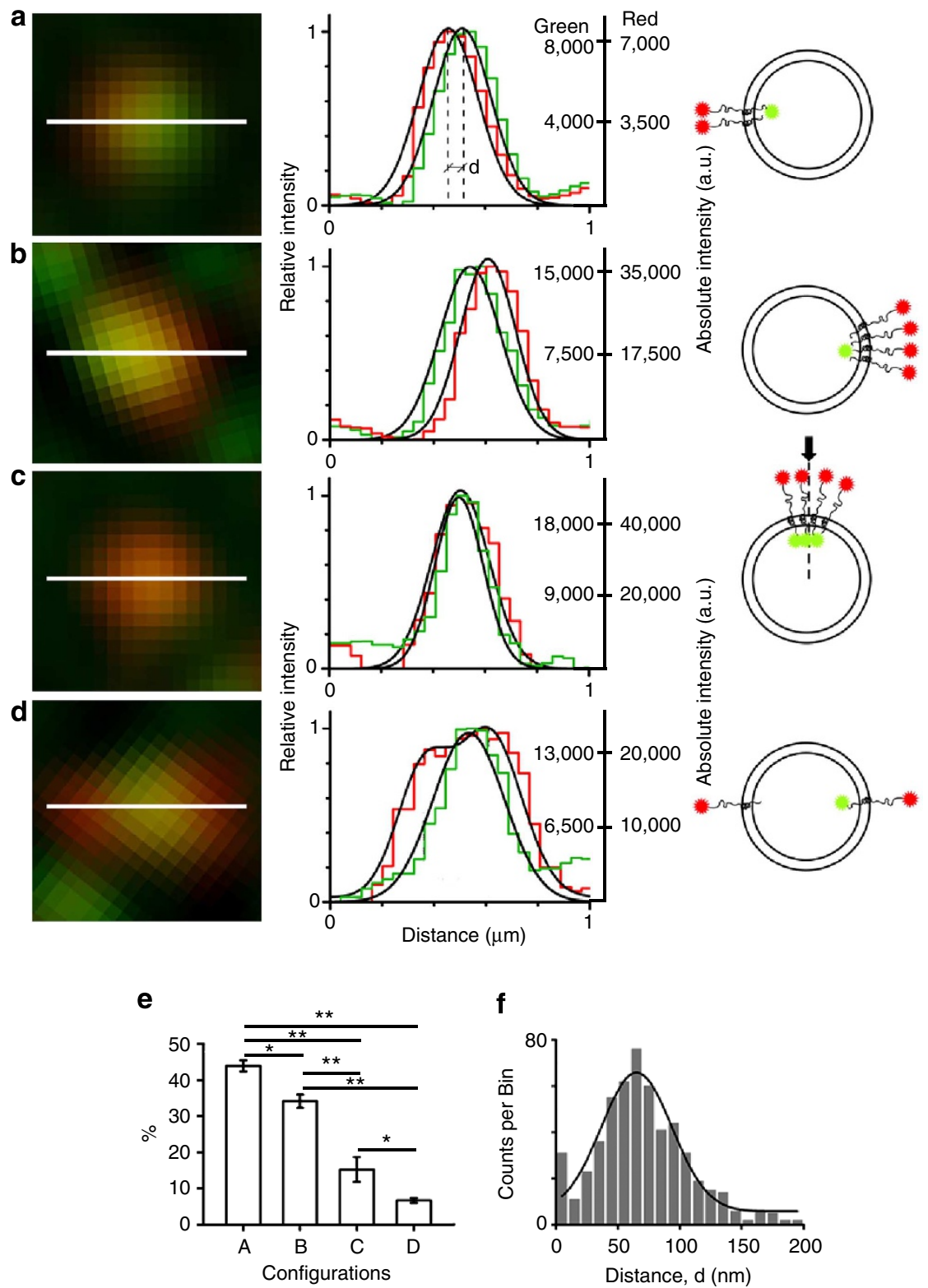

Figure 2 | Patterns of two Sb2-associated fluorophores reveal nonuniform vesicular distribution of Sb2. (a-d) Each panel contains (left to right) a SIM image, a graph and a drawing. SIM images (left column) show different patterns of Atto (red) and YpH (green) fluorophores tagging Sb2. Graphs (middle column) show normalized/relative fluorescence intensity profiles (red and green fluorescence along the section, that is, line, in corresponding SIM image). The right ordinates of the intensity profiles show absolute intensity (a.u., arbitrary unit) of green and red fluorescent puncta. Schematics in the right column show possible arrangements of two fluorophores in a single vesicle. The arrow and vertical dash-line are drawn to visualize the top view of the fluorophore pair (c). The order of images (a-d) is according to abundance (shown in \%) of the pattern observed in astrocytes as summarized in graph (e). The error bars represent s.e.m. We analyzed three astrocytes in which a total of 349 vesicles were examined. ${ }^{\star} P<0.02$ and ${ }^{\star \star} P<0.001$ (ANOVA). As indicated in line profile in $\mathbf{a}$, distance (proximity) between the green and red fluorophores, $d$, can be determined as the distance between the peaks. The frequency distribution plot of inter-fluorophore distance measured for all $\mathrm{YpH}$-Atto pairs is shown in $\mathbf{f}$. The mean value for the distance between $\mathrm{YpH}$ and Atto is $65 \pm 2 \mathrm{~nm}$ (mean \pm s.e.m.), which is calculated by fitting a Gaussian curve (f) on a frequency distribution plot of the form Counts/bin $=$ $\left.\{A /[\sigma(\sqrt{ } 2 \pi)]\} \times \exp \left\{-[\mathrm{x}-\mu)^{2} / 2 \sigma^{2}\right]\right\}$ (Equation 1) where $\mathrm{x}=$ distance $(\mathrm{nm})$, total count $A=(4,295 \pm 503) ; \sigma=28.6 ; \mu=(65 \pm 2) \mathrm{nm}$. The squared correlation coefficient $R^{2}=0.90, n=541$ vesicles, 5 cells; difference from zero was statistically significant $(P<0.001 ;$ ANOVA).

revealed that the distribution of bead and $\mathrm{YpH}$ intensity values exhibit single-mode distributions (Supplementary Fig. 3d,e, respectively), albeit $\mathrm{YpH}$, unlike bead fluorescence, is skewed from normality (D'Agostino test $P<0.01$ and $P>0.05$, respectively). Of note, each distribution here contains signals from many puncta (for comparison with individual YSpH punctum see Fig. 4b). It should be noted that the $\mathrm{YpH}$ labelling only discloses a subset of vesicles containing exogenous $\mathrm{Sb} 2$, that is, YSpH.
However, when all (endogenous and exogenous) Sb2 molecules are labelled using antibodies, there are apparent multiple modes in the Atto intensities frequency histogram (Supplementary Fig. 3f). Although this optical fluorescence intensity method does not distinguish single molecules from other unitary or quantal intensities (a finite fluorescence 'packet'), these results are consistent with the notion that $\mathrm{Sb} 2$ is non-uniformly distributed and that likely there is on average more than one unit or molecule 
a
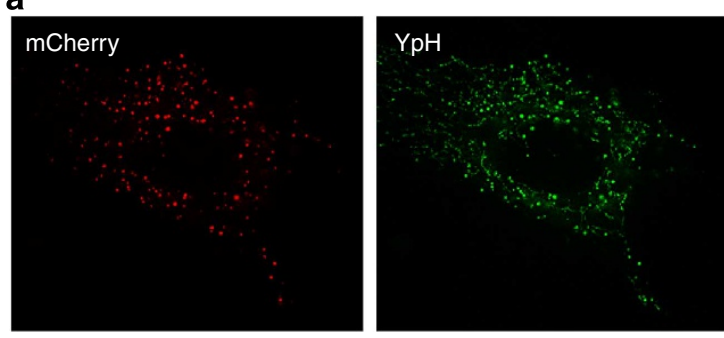

b

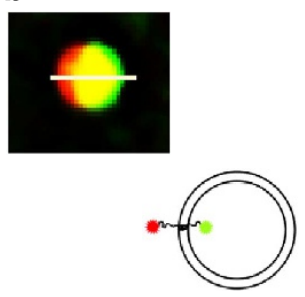

e
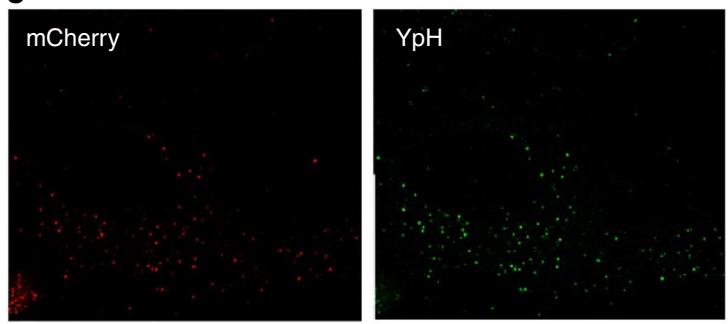

c

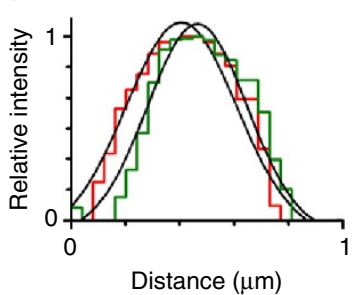

d
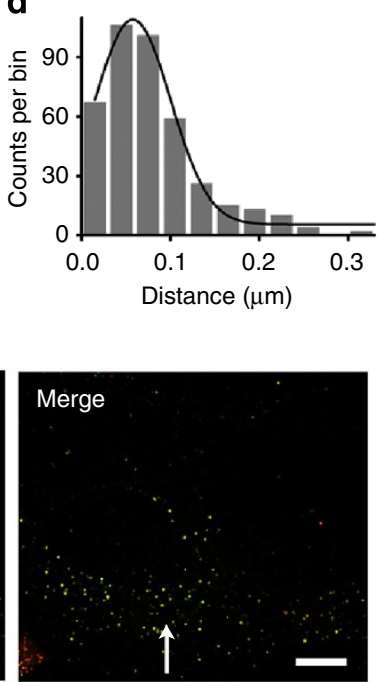

f
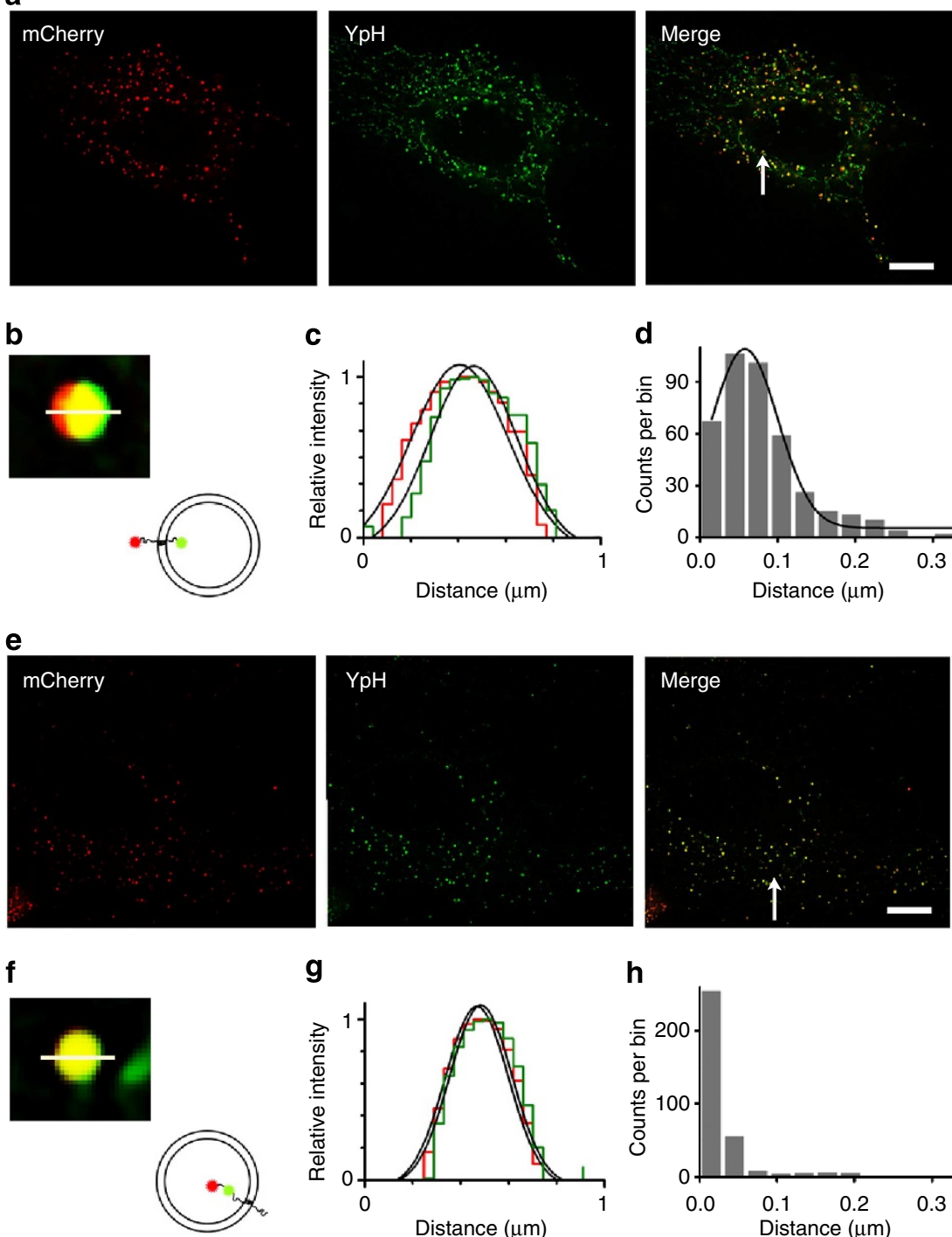

h
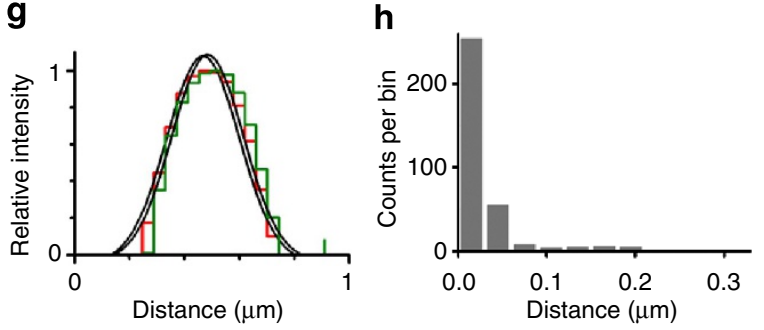

Figure 3 | SIM measurements of the distance between fluorescent proteins appended to Sb2 termini. a and e are the SIM images showing astrocytes transfected with mCherry-YSpH and $\mathrm{YSpH}-7$ aa-mCherry plasmids, respectively. Bar, $10 \mu \mathrm{m}$. Merge panels show the overlay of the fluorescence of $\mathrm{mCherry} \mathrm{(red)} \mathrm{and} \mathrm{YpH}$ (green) signals. $\mathbf{b}$ and $\mathbf{f}$ are close-up images of the mCherry and $\mathrm{YpH}$ pairs obtained from the images (Merge, arrow) in $\mathbf{a}$ and $\mathbf{e}$, respectively. The inset drawings indicate the orientation of $\mathrm{YpH}$ and $\mathrm{mCherry}$ decorating Sb2. $\mathbf{c}$ and $\mathbf{g}$ show relative fluorescence intensity profiles along the lines drawn in $\mathbf{b}$ and $\mathbf{f}$. The histograms $\mathbf{d}$ and $\mathbf{h}$ show frequency distribution plots of the distances between the two fluorophores. (d) In case of $\mathrm{mCherry}-\mathrm{YSpH}$, the mean value of the distance (peak-to-peak, as in Fig. 2a) between $\mathrm{YpH}$ (intravesicular) and mCherry (cytoplasmic) is $57 \pm 1 \mathrm{~nm}$ (mean \pm s.e.m.) $\left(R^{2}=0.98, n=403\right.$ vesicles, 7 cells; significantly different than zero, $P<0.001$; ANOVA). To measure the peak-to-peak distance, we fitted Gaussian curves using Equation 1. (h) In the case of YSpH-7aa-mCherry, the mean distance between two fluorophores juxta-positioned (spacing provided by a 7-aa linker) in the vesicular lumen is negligible ( $0 \pm 5 \mathrm{~nm} ; n=338$ vesicles, 6 cells).

of Sb2 present on the surface of a single vesicle, which we investigated further below. Of course, a fluorescent bead contains more than one molecule of dye, but as the dye loading is uniform throughout the bead as per its amorphous polymer backbone, the bead population fluorescence intensity distribution shows an appearance of a single 'packet'. In YSpH-expressing astrocytes that were immunolabelled by an anti-Sb2 antibody conjugated with Atto dye, we did not observe a correlation between the fluorescence intensity and the area of the Atto fluorescent puncta $\left(R^{2}=0.05, P=0.28\right)$.

In an attempt to determine the approximate number of $\mathrm{Sb} 2$ molecules laden in each vesicle and used in the process of vesicular fusion, using CLSM, we have monitored vesicle YSpH fluorescence in live astrocytes that were stimulated by ATP
$(0.1 \mathrm{mM})$. By activating purinergic receptors, ATP increases cytosolic $\mathrm{Ca}^{2+}$ and promotes the merger between the vesicle and the plasma membrane, leading to the formation of a fusion pore ${ }^{12,26-28}$. On fusion pore formation, alkalinization of the vesicle lumen is expected, as vesicular protons exit into the extracellular medium. This would be reflected by an increase in $\mathrm{YSpH}$ fluorescence emission. Alternatively, the vesicular lumen can be chemically alkalinized, so that, even when the fusion pore is closed, YSpH signal increases (Supplementary Fig. 1c,d). Full alkalinization of vesicles can be accomplished by the application of $\mathrm{NH}_{4} \mathrm{Cl}^{29,30}$. Hence, to assess the extent to which ATP stimulation (with the main conduit for protons to exit the vesicle lumen via $\mathrm{Ca}^{2+}$-dependent fusion pore opening) elicited vesicle alkalinization, we subsequently added $\mathrm{NH}_{4} \mathrm{Cl}(45 \mathrm{mM})$ to 

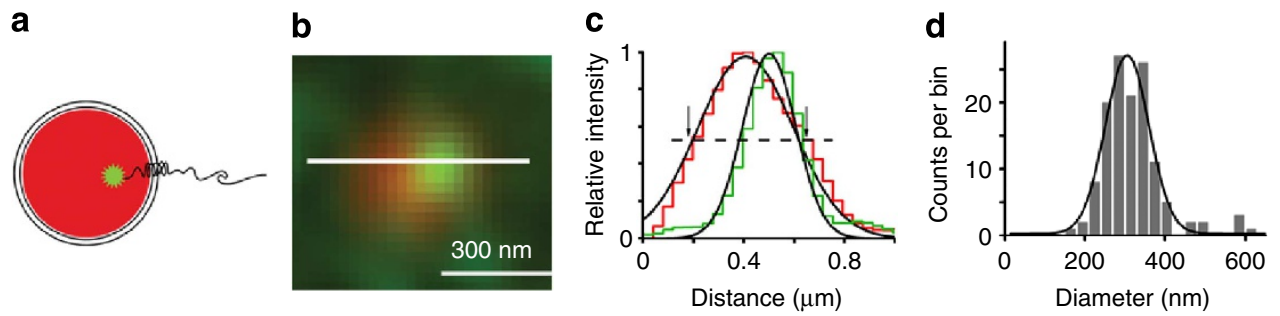

Figure 4 | Measurement of vesicle diameter in live astrocytes by SIM. (a) Diagram of an astrocytic vesicle loaded with LysoTracker DND-99 (red), expressing YSpH (green) and (b) the corresponding SIM image in a live astrocyte. Bar, $300 \mathrm{~nm}$. (c) Fluorescence intensity profile of LysoTracker and $\mathrm{YpH}$ in the vesicle lumen along the line drawn in $\mathbf{b}$. The dashed line indicates how the apparent diameter of a vesicle was determined, by measuring the FWHM of Gaussian curve of the red fluorophore. Note the intersection with Gaussian curve drawn on normalized intensity of the red fluorophore, marked with arrows. (d) The frequency distribution plot for apparent vesicle diameter, as defined in c. The mean value (mean \pm s.e.m.) of the apparent vesicle diameter is $305 \pm 3 \mathrm{~nm}\left(n=129\right.$ vesicles, 9 cells), measured by fitting a Gaussian curve of the form described in Equation $1\left(R^{2}=0.94\right.$; significantly different from zero, $P<0.001$; ANOVA).

the bath to increase vesicle lumen $\mathrm{pH}$ independent of the fusion pore formation (Fig. 5a). It should be noted that inherent to our experimental design, here we could only interrogate those vesicles that undergo kiss and run with stable pore formation of varying duration (see below), but not the full fusions, where the vesicle collapses into the plasma membrane.

The individual vesicles of astrocytes subjected to the above experimental paradigm show three types of responses (Fig. 5b): (i) ATP stimulation failed to increase YSpH fluorescence, whereas the ensuing application of $\mathrm{NH}_{4} \mathrm{Cl}$ increased $\mathrm{YSpH}$ fluorescence (to reveal the presence of chimeric Sb2), which was the most abundant pattern occurring in $\sim 60 \%$ of cases (261 of 430 vesicles; 9 cells) and likely representing failure of fusion pore opening (Fig. 5b, left); (ii) ATP caused a persistent increase in $\mathrm{YSpH}$ fluorescence intensity, which was further increased by the subsequent addition of $\mathrm{NH}_{4} \mathrm{Cl}$, a response occurring in $\sim 35 \%$ ( $n=149$ vesicles; 9 cells) of cases (Fig. $5 \mathrm{a}$ and $\mathrm{b}$, middle) and (iii) ATP evoked a transient increase in YSpH fluorescence intensity, which returned to resting levels before the increase in $\mathrm{YSpH}$ fluorescence intensity was elicited by $\mathrm{NH}_{4} \mathrm{Cl}$, an outcome occurring in $\sim 5 \%$ ( $n=20$ vesicles; 9 cells) of all cases (Fig. $5 \mathrm{~b}$, right). The latter two ATP-productive types of responses (ii and iii) likely represent cases where ATP stimulation was associated with a fusion pore opening, which was either persistently open, throughout the time-course of our approach, in the former case (ii), or opened only transiently in the latter case (iii). Such fusion pore changes have been described in astrocytes ${ }^{26}$ and in other cell types $^{31,32}$.

Next, we used the change in $\mathrm{YSpH}$ intensity measurements from individual vesicles obtained in the above described experiments to generate $\mathrm{YSpH}$ intensity distributions at resting (no vesicular fusions)/background condition, as well as during the sequential stimulation with ATP and $\mathrm{NH}_{4} \mathrm{Cl}$. Our aim was to define the putative number of $\mathrm{YSpH}$ (that is, exogenous $\mathrm{Sb} 2$ ) molecules in fusion pore forming single vesicles. We first obtained the distribution of the noise (basal level of $\mathrm{YSpH}$ ) intensity changes, which expectedly yielded an average fluorescence change near zero (Fig. 5c, left). We then obtained distributions of $\mathrm{YSpH}$ intensity changes during productive ATP stimulation (Fig. 5c, middle) and subsequent chemical alkalinization of vesicles with $\mathrm{NH}_{4} \mathrm{Cl}$ (Fig. $5 \mathrm{c}$, right). All distributions were fitted with Gaussian curves using Equation 1 (with $\mathrm{x}=\Delta \mathrm{F}$; see fitting parameters in Table 1) to obtain multimodal values for all the distributions (Fig. 5d). As one would expect, the first mode corresponds to background $\mathrm{YSpH}$ intensity changes (Fig. 5d, open bars), while the second mode represents the quantum of $\mathrm{YSpH}$ intensity change due to the stimulation, with subsequent modes being integer multiples. Surprisingly, ATP stimulation generated only one mode, that of the single quantum (Fig. $5 c$, middle and Figure $5 \mathrm{~d}$, dark grey), while $\mathrm{NH}_{4} \mathrm{Cl}$, beside the single quantum, also generated integer multiples (Fig. $5 \mathrm{c}$, right and Fig. 5d, light grey). This implicates a differential accessibility of vesicular lumen to de-protonation of $\mathrm{YpH}$ fluorophore during physiological ATP stimulation, as opposed to enforced chemical alkalinization by $\mathrm{NH}_{4} \mathrm{Cl}$ (see Discussion). Nonetheless, these modal peaks of YSpH fluorescence are distributed as multiples of fundamental quantal unit, likely representing the number of YSpH molecules within individual vesicles, as revealed by a linear relationship between the apparent number of $\mathrm{YSpH}$ molecules and change in $\mathrm{YSpH}$ intensity (Fig. 5e). Our results indicate that there are maximum of five units or molecules of $\mathrm{YSpH}$, that is, exogenous Sb2, in an astrocytic vesicle. However, only one $\mathrm{YSpH}$ molecule appeared necessary for fusion pore formation as per ATP stimulation data.

Photobleaching of $\mathrm{YpH}$ authenticates the number of exogenous $\mathbf{S b} 2$ molecules. We performed photobleaching experiments to cross-check the above determined number of YSpH molecules in a single vesicle. As above, we transfected the astrocytes to express $\mathrm{YSpH}$ and stimulated them with $\mathrm{NH}_{4} \mathrm{Cl}$ solution. The CLSM imaging was accomplished using regular (as above) laser power and detector gain settings. The bleaching of $\mathrm{YpH}$ fluorophores was done by increasing the laser power by the factor of 2-3. We observed two types of $\mathrm{YpH}$ fluorescence decay: (a) apparent exponential and (b) stepwise decays occurring in $\sim 30 \%$ and $\sim 70 \%$, respectively, out of total analyzed events $(n=277)$.

We selected the stepwise events to analyse the number of YSpH molecules. We observed different step decreases in YSpH fluorescence intensity originating from individual vesicles. Figure 6a displays three representative examples of such events: (i) a small step decrease in $\mathrm{YpH}$ fluorescence, (ii) a step decrease by the factor of two, compared with the former event and (iii) an event with three discrete step decreases in $\mathrm{YpH}$ fluorescence (Fig. 6a). By plotting the frequency distribution of the above type of events (Fig. 6b), we observed distinct modes of $\mathrm{YSpH}$ fluorescence intensity. The background intensity in this set of experiments was measured in near vicinity of vesicles $(n=108$ events). The distribution of the background fluorescence could be fitted by a Gaussian curve (Equation 1, where $\mathrm{x}=$ Intensity (a.u.); $R^{2}=0.95$, see fitting parameters in Table 2) and yielded distribution with the mean value of $2.9 \pm 0.1$ a.u (mean \pm s.e.m., Fig. 6c). Similarly, we obtained the distribution of the YSpH intensity changes due to photobleaching of $\mathrm{NH}_{4} \mathrm{Cl}$ alkalinized vesicles, which showed multi-modality (Fig. $6 \mathrm{~d} ; R^{2}=0.91$, see fitting parameters in Table 2). As one would expect, the first 
a
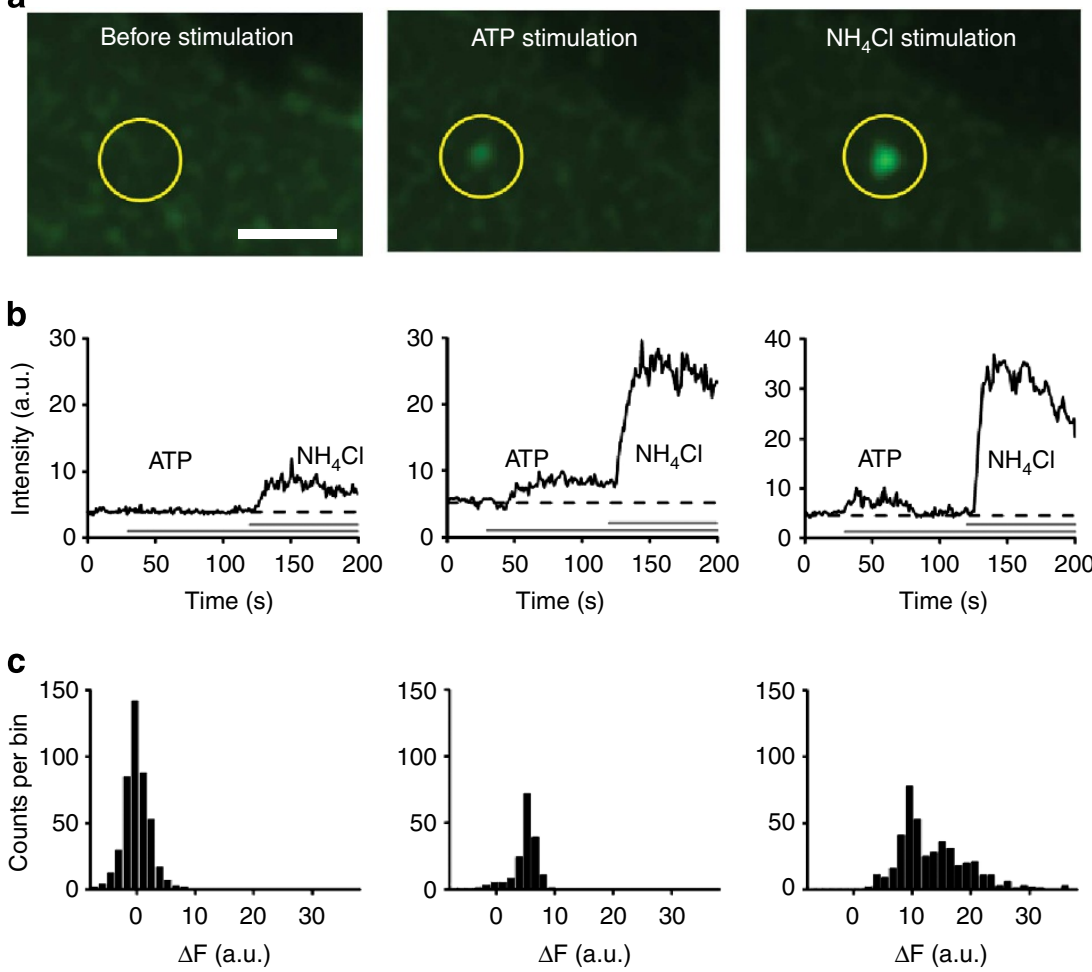

d

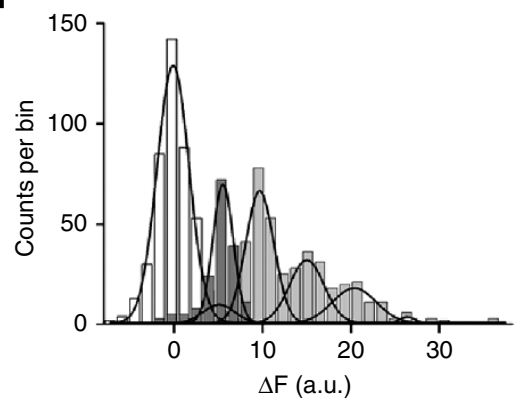

e

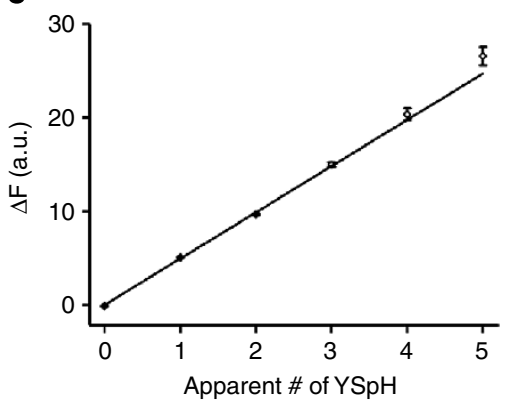

Figure 5 | Stimulation of YSpH-labelled vesicles by ATP and $\mathrm{NH}_{\mathbf{4}} \mathrm{Cl}$ reveals the number of $\mathrm{YSpH}$ molecules present on a single astrocytic vesicle.

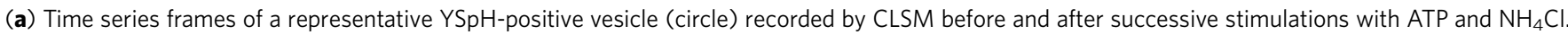
Bar, $1 \mu \mathrm{m}$. (b) Examples of time-dependent changes in the fluorescence intensity of individual vesicles: left panel, ATP failed to evoke an increase in fluorescence intensity, however, $\mathrm{NH}_{4} \mathrm{Cl}$ elicited an increase; middle panel, application of ATP elicited a sustained increase in fluorescence intensity that was augmented by $\mathrm{NH}_{4} \mathrm{Cl}$; and right panel, ATP elicited a transient increase in fluorescence intensity, which was followed with $\mathrm{NH}_{4} \mathrm{Cl}$-elicited increase. Horizontal grey bars indicate the timing of ATP/ $\mathrm{NH}_{4} \mathrm{Cl}$ applications. (c) Amplitude distributions of the change in $\mathrm{YSpH}$ fluorescence intensity $(\Delta \mathrm{F})$ recorded in nine astrocytes before and after stimulations (bin width $=1.5$ a.u.). Left panel, the change in $\mathrm{YSpH}$ intensity plot represents a background, which was obtained by monitoring changes in YSpH intensity before and after addition (at $30 \mathrm{~s}$ for $10 \mathrm{~s}$ ) of ATP (bottom horizontal line) in recordings where ATP failed to elicit a significant increase in $\mathrm{YSpH}$. Middle panel, after ATP, and, right panel, subsequent $\mathrm{NH}_{4} \mathrm{Cl}$ applications, the intensity distribution incrementally shifts to the right as vesicles alkalinize due to the pore formation (ATP) and subsequent chemical alkalinization $\left(\mathrm{NH}_{4} \mathrm{Cl}\right)$, respectively. (d) Combined plot of the change in YSpH intensity distributions for all vesicles $(n=1,045)$ from nine astrocytes: background (white) and after stimulation with ATP (dark grey) and $\mathrm{NH}_{4} \mathrm{Cl}$ (light grey). The Gaussian curves are fitted by keeping the s.d. as determined in the background shown in left panel and using Equation 1 (where $\mathrm{X}=\Delta \mathrm{F}$ (a.u.), for fitting parameters see Table 1). (e) The peaks of the Gaussian fits of different modes of intensities (obtained in $\mathbf{d}$ ) are plotted as a function of modes, that is, number of $\mathrm{YSpH}$ packets; the relationship can be described using a liner regression in the form: $\Delta \mathrm{F}($ a.u. $)=(4.95 \pm 0.04) \times$ apparent \# of $\mathrm{YSpH}+(-0.02 \pm 0.05)$, where \# is the number or multiples of $\mathrm{YSpH}$ molecules $\left(R^{2}=0.997\right.$; significantly different from zero, $\left.P<0.001 ; \mathrm{ANOVA}\right)$.

mode corresponds to background YSpH intensity changes, while the remaining four equidistant modes, report on the discrete intensities (that is, quanta), corresponding to the number of $\mathrm{YSpH}$ molecules in a single vesicle of live astrocytes (Fig. 6d; compared with Fig. 5d). This number of $\mathrm{YSpH}$ molecules obtained from photobleaching experiments validates the quantification obtained using the $\mathrm{YSpH}$ increase in fluorescence due to ATP and $\mathrm{NH}_{4} \mathrm{Cl}$ stimulation (Fig. 5e). We further analyzed exponential decays of the bleaching events of $\mathrm{YSpH}$.
The average time constant $(\tau)$ is $\sim 30 \mathrm{~s}$ ( $n=14$ vesicles), which means that on average it takes $30 \mathrm{~s}$ to bleach $63 \%$ of the total number of $\mathrm{Sb} 2$ molecules present on a vesicle, that is, three $\mathrm{YSpH}$ molecules bleach out of total five in $30 \mathrm{~s}$. Therefore, it is likely (more than $10 \%$ probability) that two $\mathrm{YSpH}$ will bleach simultaneously, especially following the start of the bleaching procedure. In agreement, the percentage of events with the double amplitude (Fig. 6a, middle) of the total bleaching events was $16.6 \%$. 


\begin{tabular}{|c|c|c|c|c|c|}
\hline $\begin{array}{l}\text { Gaussian } \\
\text { fits }\end{array}$ & $\begin{array}{c}A \\
\text { (mean } \pm \text { s.e.m.) }\end{array}$ & $\begin{array}{l}\text { Sigma } \\
(\sigma)\end{array}$ & $\begin{array}{c}\mu \\
\text { (mean } \pm \text { s.e.m.) }\end{array}$ & $R^{2}$ & $n$ \\
\hline Background & $582 \pm 19$ & 1.8 & $-0.1 \pm 0.1$ & 0.98 & 446 \\
\hline+ ATP & $200 \pm 6$ & 1.1 & $5.5 \pm 0.0$ & 0.98 & 169 \\
\hline \multicolumn{6}{|l|}{$+\mathrm{NH}_{4} \mathrm{Cl}$} \\
\hline 1st & $42 \pm 13$ & 1.6 & $5.0 \pm 0.5$ & 0.95 & 430 \\
\hline 2nd & $263 \pm 15$ & 1.4 & $9.7 \pm 0.1$ & 0.95 & 430 \\
\hline $3 r d$ & $154 \pm 31$ & 1.8 & $15.0 \pm 0.2$ & 0.95 & 430 \\
\hline 4th & $108 \pm 30$ & 2.3 & $20.4 \pm 0.6$ & 0.95 & 430 \\
\hline 5 th & $9 \pm 5$ & 0.3 & $26.7 \pm 0.9$ & 0.95 & 430 \\
\hline
\end{tabular}

The ratio between exogenous and endogenous $\mathrm{Sb} 2$ molecules. To obtain the number of endogenous Sb2 molecules present on individual astrocytic vesicles, we used Sb2 immunolabelling of astrocytes transfected to express $\mathrm{YSpH}$. Such individual astrocytes contain a mixture of vesicles, those laden with endogenous Sb2 alone (Atto positive, $\mathrm{YpH}$ negative) along with others that are dually laden with both endogenous $\mathrm{Sb} 2$ and $\mathrm{YSpH}$ (Atto and $\mathrm{YpH}$ positive) (Fig. 7a left). In addition, we compared the Sb2 immunoreactivity Atto signals of dually laden vesicles to those of native vesicles from untransfected astrocytes (Fig. 7a right). The distributions and cumulative plots of Atto fluorescence intensity of Sb2-immunolabelled vesicles that besides endogenous Sb2 (w/o YSpH; $n=262$ ) contain exogenous Sb2 in form of $\mathrm{YSpH}$ (w YSpH; $n=958$ ) show larger median values for Atto signals (Fig. 7b); the additional fluorescence of vesicles containing both endogenous and exogenous Sb2 was only $\sim 0.33$ of that found in

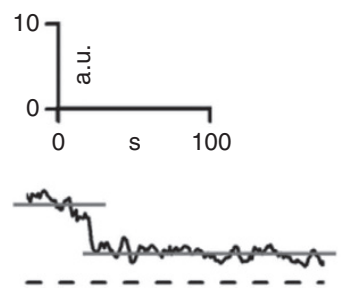

b

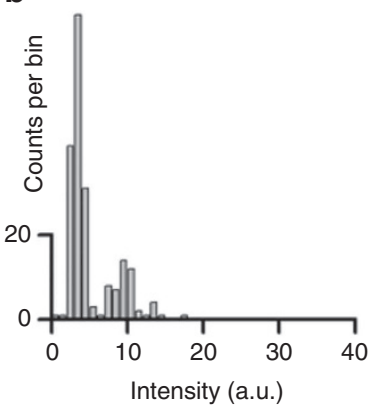

C

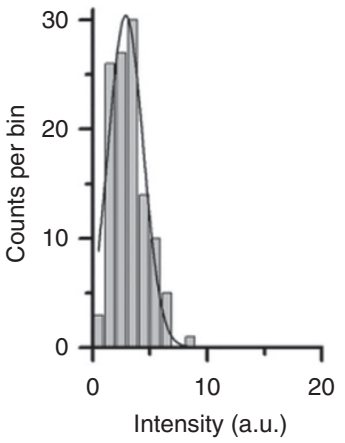

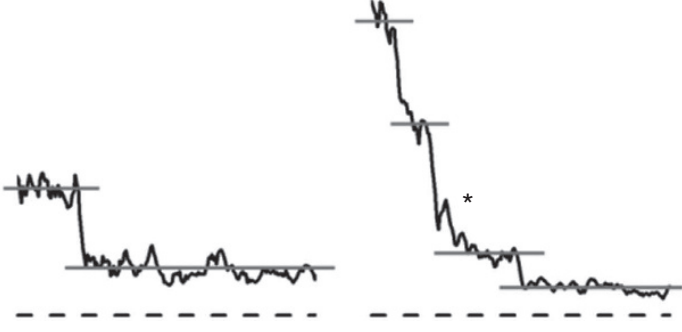
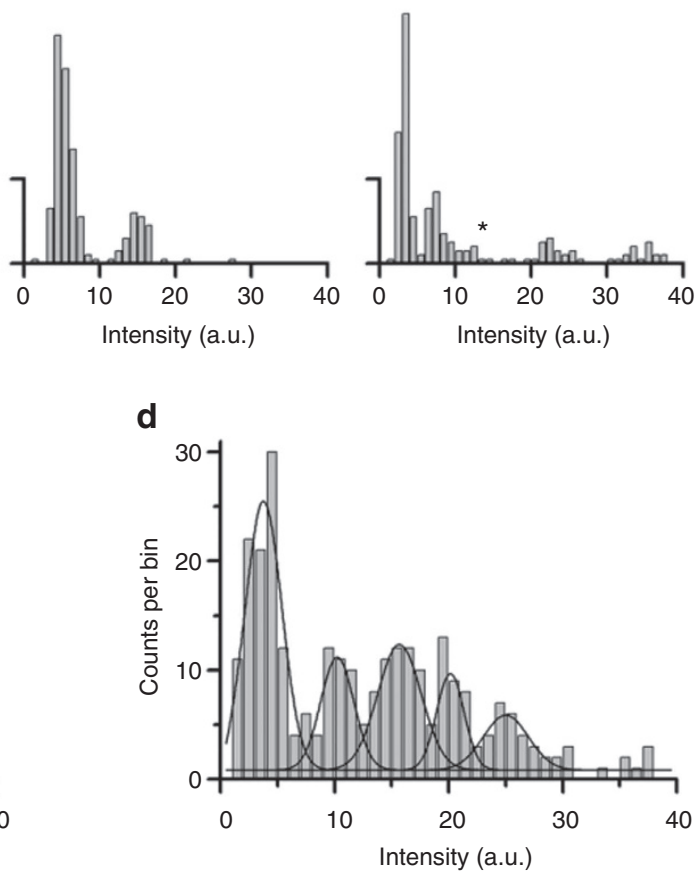

Figure 6 | Photobleaching of $\mathrm{NH}_{4} \mathrm{Cl}$-alkalinized YSpH-laden vesicles confirms the number of YSpH molecules present on a single astrocytic vesicle. (a) Examples of time-dependent changes in the fluorescence intensity signals of individual vesicles: left panel, a single step decrease in the YpH fluorescence, middle panel, a single step decrease in the $\mathrm{YpH}$ fluorescence with double amplitude, compared with the event shown in the left panel, and, right panel, multiple step decreases in $\mathrm{YpH}$ fluorescence with three discrete steps, ‘`’ denotes another step decrease, which according to frequency plot does not represent a discrete step, but rather belongs to the distribution of the final discrete step. Dashed lines represent the baseline (zero-line) and grey lines show the levels of $\mathrm{YpH}$ fluorescence signals at discrete steps. (b) The respective distributions of the fluorescence intensity amplitudes of signals shown in the panel a. The first peak in amplitude plots denotes the lowest intensity observed. (c) The intensity distribution of the background, which was obtained by measuring the fluorescence intensity of the vesicle surroundings after $180 \mathrm{~s}$ of bleaching $\left(R^{2}=0.95 ; n=108\right.$ events; bin width $=1.5$ a.u. $)$. (d) Fluorescence intensity distribution of YSpH-labelled vesicles after bleaching ( $R^{2}=0.91 ; n=277$ vesicles; bin width $=1.5$ a.u. $)$. The Gaussian curves are fitted by using Equation 1 ( $x=$ Intensity (a.u.); see fitting parameters in Table 2). 
vesicles containing only endogenous $\mathrm{Sb} 2$, indicating that for every one molecule/quantum of $\mathrm{YSpH}$ there are $\sim 3$ molecules of endogenous $\mathrm{Sb} 2$. A similar ratio was obtained when comparing $\mathrm{Sb} 2$ immunoreactivity/Atto signals from individual vesicles $(n=419)$ of untransfected astrocytes with those from dually laden vesicles $(n=499)$ of YSpH-expressing astrocytes (Fig. 7c); the additional Atto fluorescence of vesicles containing both Table 2 | Area under curve $(A)$ and mean value of each peak
$(\mu)$ obtained from Gaussian curves in bleaching experiment.

\begin{tabular}{|lccccc|}
$\begin{array}{l}\text { Gaussian } \\
\text { fits }\end{array}$ & $\begin{array}{c}\boldsymbol{A} \\
\text { (mean } \pm \text { s.e.m.) }\end{array}$ & $\begin{array}{c}\text { Sigma } \\
(\boldsymbol{\sigma})\end{array}$ & $\begin{array}{c}\boldsymbol{\mu} \\
\text { (mean } \pm \text { s.e.m.) }\end{array}$ & $\boldsymbol{R}^{\mathbf{2}}$ & $\boldsymbol{n}$ \\
\hline Background & $116 \pm 6$ & 1.5 & $2.9 \pm 0.1$ & 0.95 & 108 \\
& & & & & \\
Steps & & & & & \\
1st & $99 \pm 9$ & 1.5 & $3.8 \pm 0.1$ & 0.91 & 277 \\
2nd & $38 \pm 10$ & 1.3 & $10.2 \pm 0.3$ & 0.91 & 277 \\
3rd & $55 \pm 16$ & 1.8 & $15.7 \pm 0.4$ & 0.91 & 277 \\
4th & $26 \pm 12$ & 1.0 & $20.1 \pm 0.4$ & 0.91 & 277 \\
5th & $25 \pm 13$ & 1.9 & $25.0 \pm 0.8$ & 0.91 & 277 \\
\end{tabular}

a

w YSpH

w/o YSpH
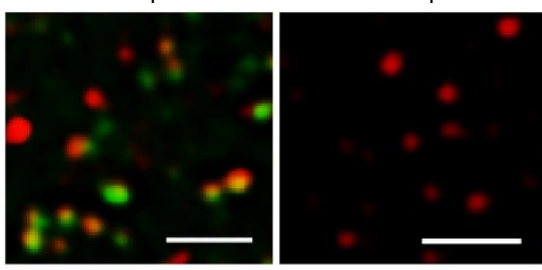

b
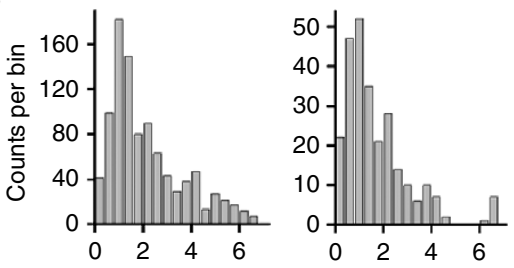

C

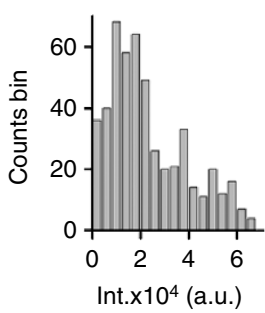

endogenous and exogenous $\mathrm{Sb} 2$ was $\sim 0.22$ of that found in endogenous Sb2-only vesicles, which translates to $\sim 5$ endogenous $\mathrm{Sb} 2$ molecules for every $\mathrm{YSpH}$ molecule.

\section{Discussion}

We have developed a new approach to label Sb2 with two different fluorescent tags and used super-resolution microscopy to visualize them in a living cell. To determine compartmentalization of proteins in vesicles of astrocytes ${ }^{33}$, we measured the length of $\mathrm{Sb} 2$ on a single vesicle in live cells in vitro. The lengths of $\mathrm{Sb} 2$ marked with $\mathrm{YpH}$ in the vesicle lumen and with a fluorescent protein (mCherry) or a dye conjugated with antibodies against Sb2 (Atto) present on the cytoplasmic site were 57 and $65 \mathrm{~nm}$, respectively. These measurements are in accordance with theoretical values of $\sim 54 \mathrm{~nm}$ and $\sim 75-80 \mathrm{~nm}$, respectively, given for the length of uncoiled $\mathrm{Sb} 2$, pHluorin, mCherry and antibodies ${ }^{18,19,21}$. However, unstructured Sb2 coils in the presence of syntaxin 1 and/or the binary acceptor syntaxin1-SNAP25 complex ${ }^{21,34}$, an expected scenario in the cellular millieu, as in our experiments. As coiled Sb2 has three $\alpha$-helices (I: $36-54$ aa, II: $77-88$ aa and III: $93-115$ aa) ${ }^{19}$ and the distance between two adjacent aa residues in the helical form d

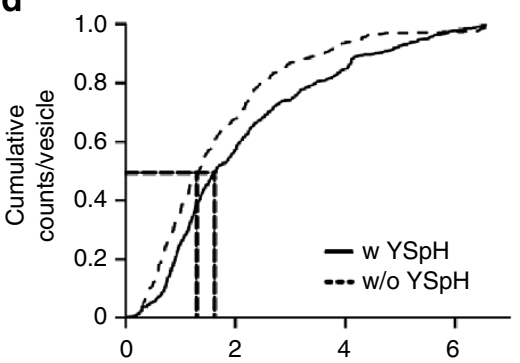

e

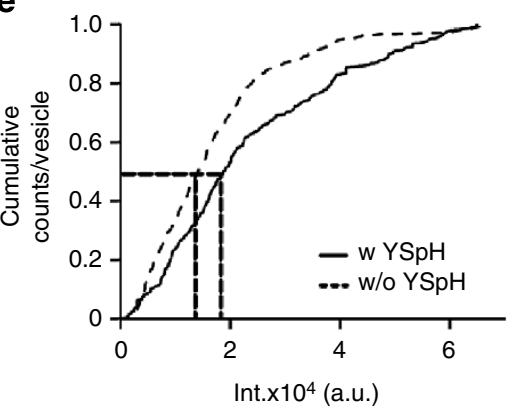

Figure 7 | Quantification of the ratio between the number of YSpH and endogenous Sb2 molecules in astrocytic vesicles. (a) Left fluorescent micrograph shows vesicles in cells transfected by $\mathrm{YSpH}$ and labelled by the Atto red dye (note that not all vesicles are labelled by both fluorophores), whereas the right panel shows vesicles in astrocytes labelled by the Atto dye only. Bar, $10 \mu \mathrm{m}$. (b,c) We obtained the distributions of Atto fluorescence intensity of Sb2 immunolabelled vesicles containing only endogenous Sb2 (w/o YSpH) and those that additionally contain exogenous Sb2 in form of $\mathrm{YSpH}(\mathrm{w} \mathrm{YSpH})$. (b) Measurements were performed on vesicles within astrocytes expressing YSpH; some vesicles contained both endogenous Sb2 and YSpH (b, left) $(n=958)$, while others were devoid of $Y S p H$, expressing only endogenous Sb2 (b, right) $(n=262)$. (d) The normalized cumulative counts of the Atto intensity in $\mathbf{b}$ show significantly different distributions $(D=0.134, P<0.001$, Kolmogorov-Smirnov test). The vertical dotted lines drawn indicate the median value of 18,772 a.u. (with $\mathrm{YSpH}$ ) and 14,115 a.u. (without $\mathrm{YSpH}$ ) for Atto signals; horizontal dotted line indicates $50 \%$ of the total vesicle population. The difference between these two median values (4,657 a.u.) likely denotes the quantum fluorescence of exogenous $\mathrm{Sb2}$, that is, YSpH, which was $\sim 1 / 3$ of that found in vesicles containing only endogenous Sb2. (c) Similar distribution as in $\mathbf{b}$, only that we measured Atto fluorescence intensities of Sb2 immunolabelled vesicles in YSpH-expressing (c, left) $(n=499)$ and in non-transfected astrocytes (c, right) ( $n=419)$, respectively. (e) The normalized cumulative counts of the Atto intensity in c show significantly different distributions ( $D=0.184, P<0.001$, Kolmogorov-Smirnov test); the median values at 16,316 a.u. and 13,382 a.u. for Atto signals of $w$ and w/o YSpH vesicles and the difference between these two median values (2,934 a.u.) suggests that exogenous Sb2 molecules represent $\sim 1 / 5$ of the number of endogenous Sb2 molecules; dotted lines as in d. 
of peptide or protein is smaller by $\sim 0.15 \mathrm{~nm}$ than in an elongated form $^{35,36}$, coiled Sb2 (containing total of 51 coiled aa) should be about $\sim 8 \mathrm{~nm}$ shorter compared with an uncoiled version. Consequently, the theoretical expectations for the above measurements then would be $\sim 47 \mathrm{~nm}$ and $\sim 67-72 \mathrm{~nm}$, values that can be readily observed in $\mathrm{YpH}$-mCherry (Fig. 3d) and $\mathrm{YpH}$ Atto (Fig. 2f) distributions around the peak measurements. Thus, it is likely that we have been observing a mixture of uncoiled and coiled Sb2; alternatively, the jitter in the measurement could be technical in nature (that is, already mentioned variability in fluorophore alignments with respect to the imaging plane).

We measured the intensity of $\mathrm{YpH}$ and Atto from SIM images and classified $\mathrm{YpH} / \mathrm{Sb} 2$ pairs into various categories based on the configurations or patterns of these fluorophores on astrocytic vesicles (Fig. 2). The most common observed pattern 'a' likely represents a vesicle in which the number of $\mathrm{Sb} 2$ molecules is relatively low, as per comparable absolute intensities of $\mathrm{YpH}$ and Atto (Fig. 2a, middle). The pattern 'b' whereby Atto fluorophore exhibited nearly three times higher fluorescence intensity than $\mathrm{YpH}$ intensity (Fig. 2b, middle) could correspond to clustering of $\mathrm{Sb} 2$, as noted previously by an X-ray study ${ }^{37}$. Previous reports indicate that $\mathrm{Sb} 2$ undergoes dimerization specifically at the transmembrane domain ${ }^{38,39}$. $\mathrm{YpH}$ and Atto pattern ' $c$ ' (Fig. 2c, middle) offers a dichotomous interpretation: (i) either the orientation of the Atto cluster is not parallel to the imaging plane or (ii) there may be two vesicles placed one over the other. The least common image observed pattern 'd' (Fig. 2d, middle) was interpreted as Atto molecules present on two extremes of a vesicle circumference, while $\mathrm{YpH}$ is situated between two Atto puncta. Although it is tempting to use the distance between two peaks of Atto signal enveloping or flanking the $\mathrm{YSpH}$ signal to coarsely estimate the vesicle diameter, such measurements could cause errors associated with size and positioning of antibodies, and we have rather developed an alternative strategy to obtain the estimate of vesicular size in live astrocytes.

Several approaches have been used to measure vesicle diameters in astrocytes ${ }^{26,40,41}$. Here we used a distinct approach based on Sb2 tagging in conjunction with dye filling of the vesicular lumen. After staining acidic organelles with LysoTracker, we obtained the apparent diameters of selected YSpH-laden vesicles as $305 \pm 4 \mathrm{~nm}$ in live astrocytes. This is likely a slight overestimation due to point-spread-function convolution, as seen in control experiments estimating the size of fluorescent beads of the known diameter. However, these measurements are in good agreement with measurements of vesicles tagged with $\mathrm{Sb} 2$ in live astrocytes investigated using evanescence wave microscopy; those vesicles showed an apparent diameter of $\sim 312 \mathrm{~nm}$ (ref. 26). For our present measurements, only a subset of vesicles was interrogated due to the fact that a vesicle expressing a visible $\mathrm{YSpH}$ requires optimized $\mathrm{pH}$ in the vesicle lumen along with co-labelling with LysoTracker. Namely, LysoTracker stains mostly acidic compartments of astrocytes ${ }^{25}$, whereas $\mathrm{YSpH}$ becomes fluorescent once the fusion pore is open or by other mechanisms that lead to alkalinization.

Some published reports provide information about the number of $\mathrm{Sb} 2$ proteins or SNARE complexes present on a vesicle membrane or number/ratio involved in the fusion of a vesicle membrane with the plasma membrane in neurons ${ }^{5,42-46}$. On the basis of changes in $\mathrm{YSpH}$ fluorescence, we estimated the number of $\mathrm{YSpH}$ molecules present in an astrocytic vesicle, as well as the number of $\mathrm{YSpH}$ molecules involved in fusion pore formation.

The intensity patterns after application of ATP or $\mathrm{NH}_{4} \mathrm{Cl}$ were found to be different, indicating that the addition of ATP makes visible only some $\mathrm{YSpH}$ molecules due to dequenching mediated by partial and/or by restricted alkalinization of the vesicle lumen after opening of the narrow fusion pore. This is an intriguing finding as it may point out to an unappreciated mechanism for $\mathrm{pH}$ buffering of vesicular lumen and it should be explored in the near future. Alternatively, there could be a subpopulation of vesicles at the membrane that fuses when astrocytes are stimulated with ATP or vesicles are clustered, and only some have access to the extracellular alkaline medium or there may be a $\mathrm{pH}$ gradient in the vesicle lumen in case of comparable fluxes through pumps and the fusion pore. Nonetheless, addition of $\mathrm{NH}_{4} \mathrm{Cl}$ makes all the content of the vesicle lumen alkaline because ammonia in equilibrium with ammonium ions in solution diffuses across cell membranes and increases the $\mathrm{pH}^{47}$. Therefore, in single-vesicle experiments, $\mathrm{pH}$ change by ATP elicits fluorescence of a limited portion of all available luminal vesicle $\mathrm{pH}$-sensitive fluorescent proteins or a limited number of vesicles in a cluster. The amplitude distribution plot for ATP shows a single mode (Fig. $5 c$, middle). The amplitude distribution plot for $\mathrm{NH}_{4} \mathrm{Cl}$ with multiple modes (Fig. 5c, right) signifies that after chemical alkalinization, all units of $\mathrm{YSpH}$ present in a vesicle lumen are fluorescent. If the modal peaks represent units or molecules, then these should represent simple integer multiples. Indeed, the mean of Gaussian distributions are equidistant with a value of 5 a.u., representing the fundamental quantal unit, which indicates the apparent number of $\mathrm{YSpH}$ units or molecules present in a single vesicle. The results show that there is at least one and not more than five YSpH (exogenous Sb2) units or molecules present on an astrocytic vesicle (Fig. 5d). On the basis of a single-mode distribution during ATP stimulation, it is most likely that only one $\mathrm{YSpH}$ molecule is involved in initial fusion pore formation during the merger between vesicle and plasma membranes in astrocytes. Indeed, these measurements were limited to kiss-and-run events as full fusion would cause dissipation of $\mathrm{YSpH}$ signal in the plasma membrane as vesicular membrane collapses to and dilutes its content within the plasma membrane ${ }^{26}$. The time-dependent intensity profile for each fluorescent punctum, present in a cell after stimulation with ATP and $\mathrm{NH}_{4} \mathrm{Cl}$, demonstrated three different types of events (Fig. 5b). The most abundant event type in an astrocyte is an $\mathrm{NH}_{4} \mathrm{Cl}$-elicited increase in $\mathrm{YSpH}$ fluorescence preceded by the failure of ATP stimulation to evoke such a response. ATP influences two types of events: one shows a step increase in fluorescent intensity after ATP that is further enhanced by the addition of $\mathrm{NH}_{4} \mathrm{Cl}$ and another type of event also shows a step increase after ATP but, after approximately $1 \mathrm{~min}$, it returns to the initial intensity, which then increases with the addition of $\mathrm{NH}_{4} \mathrm{Cl}$. This rare event may be due to the fact that a secretory vesicle situated close to the plasma membrane can form a stable fusion pore $^{48}$.

We also obtained a comparable estimated number of $\mathrm{YSpH}$ molecules using photobleaching of alkalinized YSpH-laden vesicles. Thus, the estimated numbers of exogenous Sb2 molecules per vesicle in live astrocytes (Figs 5 and 6) appear similar using two approaches (ATP or $\mathrm{NH}_{4} \mathrm{Cl}$-mediated $\mathrm{YSpH}$ increase and photobleaching of $\mathrm{NH}_{4} \mathrm{Cl}$-alkalinized $\mathrm{YSpH}$-laden vesicles) that cross-check each other (that is, three to five exogenous $\mathrm{Sb} 2$ molecules). Furthermore, we determined the ratio of endogenous $\mathrm{Sb} 2$ to $\mathrm{YSpH}$ molecules per vesicle, leading us to conclude that individual astrocytic vesicles contain $\sim 15-25$ molecules of Sb2, which is $\sim 3-5$ times smaller amount than the previously reported value of $\sim 70$ copies of this SNARE protein per individual neuronal, that is, synaptic vesicle ${ }^{42}$.

In summary, the results provide a new insight into the nano architecture of Sb2 on an astrocytic vesicle, which is playing a role in vesicle fusion and gliotransmitter release from astrocytes.

\section{Methods}

Cell culture. Cortical astrocytes from 3-day-old female rats used in the experiments were isolated as described ${ }^{49}$. The care for experimental animals and the 
euthanization of animals were carried out in strict accordance with the following ethical codes and directives: The International Guiding Principles for Biomedical Research Involving Animals developed by the Council for International Organizations of Medical Sciences and the Directive on Conditions for Issue of License for Animal Experiments for Scientific Research Purposes (Official Gazette of the Republic of Slovenia 40/85, 22/87, 43/07). The protocol for the euthanization of the animals used in our study was approved by the Veterinary Administration of the Ministry for Agriculture and the Environment of the Republic of Slovenia (permit No: 34401-29/2009/2), issued on 22.4.2009; procedures were also in strict accordance with the National Institutes of Health Guide for Care and Use of Laboratory Animals and were approved by the University of Alabama, Birmingham Institutional Animal Care and Use Committee.

Astrocytes were plated on poly-L-lysine-coated coverslips and cultured with Dulbecco's modified Eagle medium (DMEM, high glucose content: 4,500 $\mathrm{mgl}^{-1}$ ) supplemented with $10 \%$ fetal bovine serum (FBS), $1 \mathrm{mM}$ sodium pyruvate, $2 \mathrm{mM}$ L-glutamine and $25 \mathrm{\mu g} \mathrm{ml}^{-1}$ penicillin-streptomycin antibiotics in $95 \%$ air and $5 \%$ $\mathrm{CO}_{2}$ atmosphere at $37^{\circ} \mathrm{C}$. Unless stated otherwise, all chemicals were obtained from Merck (Darmstadt, Germany) and Sigma (Diesenhofen, Germany). For experiments in Supplementary Fig. 1, cultured purified primary rat astrocytes were obtained from visual cortices of 1- to 2-day-old Sprague-Dawley rats ${ }^{8}$.

Plasmid construction. Green superecliptic synaptopHluorin (GSpH), a fluorescent $\mathrm{Sb} 2$ derivative, is a fusion protein chimera consisting of mouse $\mathrm{Sb} 2$ and modified EGFP, pHluorin, fused to Sb2 C terminus inside the vesicle lumen. On the basis of known amino acids contributing to chromophore pockets of EGFP versus $\mathrm{EYFP}^{50,51}$, we introduced mutations to GSpH to generate its yellow-shifted emission variant. This $\mathrm{YSpH}$ was constructed by polymerase chain reaction (PCR) mutagenesis using the original GSpH plasmid generously provided by Dr James E. Rothman (Yale University, New Haven, CT) ${ }^{17,52}$. Four primers were utilized containing mutations as well as 15-bp overlapping sequences for use with the InFusion 2 system (Clontech Laboratories, Mountainview, CA). PCR for both vector and insert fragments (listed below) was performed on a GSpH template introducing the desired mutations, and the resulting fragments were rejoined with InFusion 2 enzyme and buffers according to the manufacturer's instructions. The resulting plasmid $(P Y S p H)$ was sequenced and expressed in rat primary astrocyte culture using cytomegalovirus immediate early promoter contained in plasmid (Supplementary Fig. 1 for details).

Vector primers

Fwd 5' -TACCAGACAACCATTACCTGTTTTACACTTCTACTC-3'

Rev $5^{\prime}$-AGCATTGCAGACCATAGCCGAAAGTAG-3'

Insert primers

Fwd 5'-ATGGTCTGCAATGCTTTGCCAGATAC- ${ }^{\prime}$

Rev $5^{\prime}$-AGTGTAAAACAGGTAATGGTTGTCTG- $3^{\prime}$

Using the InFusion 2 subcloning system, we made two additional plasmids, one encoding YSpH additionally tagged by the red fluorescent protein mCherry on the cytoplasmic vesicle site (PmCherry-YSpH) and the other (PYSpH-7aa-mCherry) encoding the chimeric protein YSpH-7aa-mCherry in which mCherry was appended to the $\mathrm{C}$ terminus of $\mathrm{YSpH}$ via a 7 -aa linker (see Supplementary Fig. 2 for plasmid maps). PCR for the vectors was performed on the YSpH template, while the PCR for the mCherry insert fragments was performed on the pcDNA3.1/ VChR1-mCherry plasmid, kindly provided by Dr Karl Deisseroth (Stanford University, Stanford, CA $)^{4}$. The DNA sequence $\left(5^{\prime}\right.$-tcaggtctacgatctagagct- $\left.3^{\prime}\right)$ of the polypeptide linker (SGLRSRA), as we previously used ${ }^{5}$, was introduced by primer overhangs. Plasmid constructs were checked by restriction enzyme digestion and sequencing before expression in primary astrocyte culture. Primers used in subcloning are listed below.

mCherry-YSpH Vector Primers:

Fwd 5'-TGTACAAGTCGGCTACCGCTGCCACCGTC-3'

Rev 5'-CTTGCTCACCATGGTGGCACGCGTGAATTC- ${ }^{\prime}$

mCherry-YSpH Insert Primers:

Fwd 5'-ACCATGGTGAGCAAGGGCGAGGAG- $3^{\prime}$

Rev 5'-TAGCCGACTTGTACAGCTCGTCCATG-3'

YSpH-7aa-mCherry Vector Primers:

Fwd 5'-TAATCTAGAGTCGACCCGGGCGGCCGCTTCCCTTTAG-3' Rev 5'-AGATCGTAGACCTGAACCGGTTTTGTATAGTTCATCCATGCC ATG-3'

YSpH-7aa-mCherry Insert Primers:

Fwd 5'-TCAGGTCTACGATCTAGAGCTGTGAGCAAGGGCGAG-3'

Rev $5^{\prime}$-GTCGACTCTAGATTACTTGTACAGCTCGTCCATG-3'.

Plasmid DNA transfection. Astrocytes were transfected with $1-2 \mu \mathrm{g}$ of $\mathrm{pDNA}^{6,7}$ using FuGENE 6 transfecting reagent (Roche, Mannheim, Germany) and the culture medium as above (without FBS). UltroserG (3\%; Life Technologies) was added after an incubation period of $3 \mathrm{~h}$ at $37^{\circ} \mathrm{C}$, and the cells were then incubated overnight. The transfected astrocytes were examined or immunolabelled after at least $24 \mathrm{~h}$ incubation. In a subset of experiments (Supplementary Fig. 1), astrocytes were transfected with plasmids using TransIT 293 transfection reagent (Mirus Bio, Madison, WI $)^{8}$.
Immunocytochemistry. The transfected astrocytes were washed with phosphatebuffered saline (PBS), fixed in 4\% formaldehyde (Thermo Scientific, USA) in PBS for $15 \mathrm{~min}$ and incubated in $0.1 \%$ Triton X-100 for $10 \mathrm{~min}$ at room temperature. Nonspecific background staining was reduced by blocking buffer containing $3 \%$ bovine serum albumin (BSA) and $10 \%$ goat serum in PBS $\left(37^{\circ} \mathrm{C}, 1 \mathrm{~h}\right)$. We used mouse polyclonal primary antibody raised against a synthetic peptide corresponding to amino acids 1-18 of Sb2 (1:500; Abcam, Cat. No. ab3347). Astrocytes were incubated with an anti-Sb2 antibody diluted in $3 \%$ BSA in PBS for $2 \mathrm{~h}$ at $37^{\circ} \mathrm{C}$ or overnight at $4{ }^{\circ} \mathrm{C}$. The cells were then stained with secondary antibodies against mouse IgG conjugated to Atto 594 (1:100; Sigma Aldrich) at $37^{\circ} \mathrm{C}$ for $45 \mathrm{~min}$. The cells were again rinsed four times with PBS and mounted on glass slides using SlowFade Gold antifade reagent (Invitrogen, Eugene, OR).

Optical imaging. CLSM: Cells were examined with an inverted Zeiss LSM 510 META microscope (Germany) with a $63 \times / 1.4$ Oil DIC objective, He/Ne and argon lasers. YSpH was excited by a $488 \mathrm{~nm}$ line. The light emitted was collected through a band-pass filter $(505-530 \mathrm{~nm})$. SIM: The detailed architecture of astrocytic vesicles was examined using a Zeiss ELYRA PS.1 super-resolution microscope (Germany) equipped with five different grating frequencies for SIM for optimal matching of the illumination pattern to the laser wavelength and objective lens. The cells were scanned using a Plan-Apochromat $63 \times / 1.4$ Oil DIC M27 objective and HBO X-Cite 120 lamp. Cells were illuminated with laser lines (561 and $488 \mathrm{~nm}$ ). Sixteen-bit images were acquired with an EMCCD camera (Andor iXon 885) with variable exposure. The diameter of standard beads used in the intensity analysis was $200 \mathrm{~nm}$ (Zeiss, Germany). Additional details for the imaging used in a subset of Supplementary Fig. 1 can be found in Supplementary Methods.

Single-vesicle pHluorin experiments. Astrocytes expressing $\mathrm{YSpH}$ were scanned using an LSM 510 META microscope. The cells were kept in $200 \mu \mathrm{l}$ of extracellular solution for live imaging, consisting of (in $\mathrm{mM}$ ) $130 \mathrm{NaCl}, 5 \mathrm{KCl}, 2 \mathrm{CaCl}_{2}, 1 \mathrm{MgCl}_{2}$, 10 D-glucose, $10 N$-2-hydroxyethylpiperazine- $N^{\prime}$-2-ethanesulphonic acid (HEPES) $\mathrm{pH} 7.2$ and $\sim 300 \mathrm{mOsm} \mathrm{kg}^{-1}$. To increase the free concentration of calcium ions $\left(\left[\mathrm{Ca}^{2+}\right]_{\mathrm{i}}\right)$ in the cytoplasm, astrocytes were stimulated with $0.1 \mathrm{mM}$ ATP. Extracellular solution containing $45 \mathrm{mM} \mathrm{NH}_{4} \mathrm{Cl}$ was used for the vesicle alkalinization A time-lapse imaging series of 200 frames $\left(1\right.$ frame $\left.^{-1}\right)$ was acquired. During acquisition, we applied ATP and $\mathrm{NH}_{4} \mathrm{Cl}$ at $30 \mathrm{~s}$ and $120 \mathrm{~s}$, respectively, and left until the end of experiments. In photobleaching experiments, a time-lapse imaging series of 300 frames $\left(1\right.$ frame s$^{-1}$ ) was acquired. During acquisition, we applied $\mathrm{NH}_{4} \mathrm{Cl}$ at $10 \mathrm{~s}$ and increased the laser power by factor of $2-3$ after $50 \mathrm{~s}$ and both left until the end of experiments.

To stain the vesicle lumen for measurement of vesicle diameter, transfected astrocytes were incubated with $100 \mathrm{nM}$ LysoTracker Red DND-99 (Molecular Probes) at $37^{\circ} \mathrm{C}$ for $5 \mathrm{~min}$.

Image and data analysis. To quantitatively analyse large collections of images, we developed a custom tool ColocAna (Celica, Slovenia) using Matlab (Mathworks, Natick, MA). The colocalization of Atto and $\mathrm{YpH}$ was analyzed, as reported (Kreft et al., ${ }^{33}$ ), by using ColocAna software that counts red, green and colocalized pixels. The colocalization was calculated for all pixels above the threshold, which was set at $20 \%$ of maximum green and red fluorescence intensity and was expressed as the percentage of colocalized green pixels. The distance between two fluorophores is measured using a ZEN lite software (Carl Zeiss) by drawing a line profile on two different fluorophores in each of Atto- $\mathrm{YpH}$ and mCherry-YpH pairs. To measure the diameter of astrocytic vesicles, we calculated the average values of FWHM of the line profile drawn for a LysoTracker-stained vesicle ${ }^{53}$; for better precision, we drew crosshair line profiles in the $x$ and $y$ directions of the 2D plane of each punctum. Levenberg-Marquadt $X^{2}$ minimization for nonlinear least-square fitting was performed in OriginLab Pro. 8 software to fit the Gaussian curve on the histograms. The intensity analysis for the pHluorin experiments was done with ImageJ 1.35j software (National Institutes of Health, USA) by selecting square regions of interest $\left(1.33 \times 1.33 \mu \mathrm{m}^{2}\right)$ overlaid on each fluorescent punctum, and thereby mean intensities were estimated using the intensity versus time monitor plugin.

Statistical analysis. The values for the Gaussian fit are given as mean \pm s.e.m. Statistical significance of the data was analyzed in the SigmaPlot version 11 software. $P$-values $<0.05$ were considered to be statistically significant.

\section{References}

1. Nedergaard, M., Ransom, B. \& Goldman, S. A. New roles for astrocytes: redefining the functional architecture of the brain. Trends Neurosci. 26, 523-530 (2003).

2. Parpura, V. \& Zorec, R. Gliotransmission: exocytotic release from astrocytes. Brain Res. Rev. 63, 83-92 (2010)

3. Halassa, M. M., Fellin, T. \& Haydon, P. G. The tripartite synapse: roles for gliotransmission in health and disease. Trends Mol. Med. 13, 54-63 (2007).

4. Wienisch, M. \& Klingauf, J. Vesicular proteins exocytosed and subsequently retrieved by compensatory endocytosis are nonidentical. Nat. Neurosci. 9, 1019-1027 (2006). 
5. Hua, Y. et al. A readily retrievable pool of synaptic vesicles. Nat. Neurosci. 14, 833-839 (2011)

6. Jordan, R., Lemke, E. A. \& Klingauf, J. Visualization of synaptic vesicle movement in intact synaptic boutons using fluorescence fluctuation spectroscopy. Biophys. J. 89, 2091-2102 (2005).

7. Gucek, A., Vardjan, N. \& Zorec, R. Exocytosis in astrocytes: transmitter release and membrane signal regulation. Neurochem. Res. 37, 2351-2363 (2012).

8. Montana, V., Ni, Y., Sunjara, V., Hua, X. \& Parpura, V. Vesicular glutamate transporter-dependent glutamate release from astrocytes. J. Neurosci. 24, 2633-2642 (2004).

9. Verderio, C. et al. TI-VAMP/VAMP7 is the SNARE of secretory lysosomes contributing to ATP secretion from astrocytes. Biol. Cell 104, 213-228 (2012).

10. Jeftinija, S. D., Jeftinija, K. V., Stefanovic, G. \& Liu, F. Neuroligand-evoked calcium-dependent release of excitatory amino acids from cultured astrocytes. J. Neurochem. 66, 676-684 (1996).

11. Parpura, V., Fang, Y., Basarsky, T., Jahn, R. \& Haydon, P. G. Expression of synaptobrevin II, cellubrevin and syntaxin but not SNAP-25 in cultured astrocytes. FEBS Lett. 377, 489-492 (1995).

12. Stenovec, M. et al. $\mathrm{Ca}^{2+}$-dependent mobility of vesicles capturing antiVGLUT1 antibodies. Exp. Cell. Res. 313, 3809-3818 (2007).

13. Pangrsic, T. et al. Exocytotic release of ATP from cultured astrocytes. J. Biol. Chem. 282, 28749-28758 (2007).

14. Abbe, E. Beiträgezurtheorie des mikroskops und der mikroskopischenwahrnehmung. Archiv. für mikroskopische Anatomie 9, 413-418 (1873).

15. Huang, B., Bates, M. \& Zhuang, X. Super-resolution fluorescence microscopy. Annu. Rev. Biochem. 78, 993-1016 (2009).

16. Gustafsson, M. G. Surpassing the lateral resolution limit by a factor of two using structured illumination microscopy. J. Microsc. 198, 82-87 (2000).

17. Miesenbock, G., De Angelis, D. A. \& Rothman, J. E. Visualizing secretion and synaptic transmission with $\mathrm{pH}$-sensitive green fluorescent proteins. Nature 394, 192-195 (1998)

18. Rizzo, M. A., Davidson, M. W. \& Piston, D. W. Fluorescent protein tracking and detection: applications using fluorescent proteins in living cells. Cold Spring Harb. Protoc. doi:10.1101/pdb.top64 (2009).

19. Ellena, J. F. et al. Dynamic structure of lipid-bound synaptobrevin suggests a nucleation-propagation mechanism for trans-SNARE complex formation. Proc. Natl Acad. Sci. USA 106, 20306-20311 (2009).

20. Ainavarapu, S. R. et al. Contour length and refolding rate of a small protein controlled by engineered disulfide bonds. Biophys. J. 92, 225-233 (2007).

21. Sutton, R. B., Fasshauer, D., Jahn, R. \& Brunger, A. T. Crystal structure of a SNARE complex involved in synaptic exocytosis at 2.4 A resolution. Nature 395, 347-353 (1998).

22. Labrijn, A. F. et al. Access of antibody molecules to the conserved coreceptor binding site on glycoprotein gp120 is sterically restricted on primary human immunodeficiency virus type 1. J. Virol. 77, 10557-10565 (2003).

23. Squire, A., Verveer, P. J., Rocks, O. \& Bastiaens, P. I. Red-edge anisotropy microscopy enables dynamic imaging of homo-FRET between green fluorescent proteins in cells. J. Struct. Biol. 147, 62-69 (2004).

24. Shroff, H. et al. Dual-color superresolution imaging of genetically expressed probes within individual adhesion complexes. Proc. Natl Acad. Sci. USA 104, 20308-20313 (2007).

25. Potokar, M. et al. Intermediate filaments attenuate stimulation-dependent mobility of endosomes/lysosomes in astrocytes. Glia 58, 1208-1219 (2010).

26. Malarkey, E. B. \& Parpura, V. Temporal characteristics of vesicular fusion in astrocytes: examination of synaptobrevin 2-laden vesicles at single vesicle resolution. J. Physiol. 589, 4271-4300 (2011).

27. Neary, J. T., van Breemen, C., Forster, E., Norenberg, L. O. B. \& Norenberg, M. D. ATP stimulates calcium influx in primary astrocyte cultures. Biochem. Biophys. Res. Commun. 157, 1410-1416 (1988).

28. Jeremic, A., Jeftinija, K., Stevanovic, J., Glavaski, A. \& Jeftinija, S. ATP stimulates calcium-dependent glutamate release from cultured astrocytes. J. Neurochem. 77, 664-675 (2001).

29. Antonenko, Y. N., Pohl, P. \& Denisov, G. A. Permeation of ammonia across bilayer lipid membranes studied by ammonium ion selective microelectrodes. Biophys. J. 72, 2187-2195 (1997).

30. Sankaranarayanan, S. \& Ryan, T. A. Real-time measurements of vesicle-SNARE recycling in synapses of the central nervous system. Nat. Cell Biol. 2, 197-204 (2000).

31. Vardjan, N., Stenovec, M., Jorgacevski, J., Kreft, M. \& Zorec, R. Elementary properties of spontaneous fusion of peptidergic vesicles: fusion pore gating. J. Physiol. 585, 655-661 (2007).

32. Vardjan, N., Jorgacevski, J. \& Zorec, R. Fusion pores, SNAREs, and exocytosis. Neuroscientist 19, 160-174 (2013).

33. Kreft, M., Milisav, I., Potokar, M. \& Zorec, R. Automated high through-put colocalization analysis of multichannel confocal images. Comput. Meth. Program Biomed. 74, 63-67 (2004).

34. Hazzard, J., Sudhof, T. C. \& Rizo, J. NMR analysis of the structure of synaptobrevin and of its interaction with syntaxin. J. Biomol. NMR 14, 203-207 (1999).
35. Lehninger, A. L. Biochemistry: the Molecular Basis of Cell Structure and Function (Worth Publishers, 1975).

36. Stryer, L. Biochemistry (W.H. Freeman, 1995).

37. Castorph, S. et al. Structure parameters of synaptic vesicles quantified by smallangle X-ray scattering. Biophys. J. 98, 1200-1208 (2010).

38. Laage, R. \& Langosch, D. Dimerization of the synaptic vesicle protein synaptobrevin (vesicle-associated membrane protein) II depends on specific residues within the transmembrane segment. Eur. J. Biochem. 249, 540-546 (1997).

39. Fleming, K. G. \& Engelman, D. M. Computation and mutagenesis suggest a right-handed structure for the synaptobrevin transmembrane dimer. Proteins 45, 313-317 (2001).

40. Potokar, M., Stenovec, M., Kreft, M., Kreft, M. E. \& Zorec, R. Stimulation inhibits the mobility of recycling peptidergic vesicles in astrocytes. Glia 56, 135-144 (2008).

41. Bergami, M. et al. Uptake and recycling of pro-BDNF for transmitter-induced secretion by cortical astrocytes. J. Cell Biol. 183, 213-221 (2008).

42. Takamori, S. et al. Molecular anatomy of a trafficking organelle. Cell 127, 831-846 (2006).

43. van den Bogaart, G. et al. One SNARE complex is sufficient for membrane fusion. Nat. Struct. Mol. Biol. 17, 358-364 (2010).

44. Karatekin, E. et al. A fast, single-vesicle fusion assay mimics physiological SNARE requirements. Proc. Natl Acad. Sci. USA 107, 3517-3521 (2010).

45. Domanska, M. K., Kiessling, V., Stein, A., Fasshauer, D. \& Tamm, L. K. Single vesicle millisecond fusion kinetics reveals number of SNARE complexes optimal for fast SNARE-mediated membrane fusion. J. Biol. Chem. 284, 32158-32166 (2009).

46. Mohrmann, R., de Wit, H., Verhage, M., Neher, E. \& Sorensen, J. B. Fast vesicle fusion in living cells requires at least three SNARE complexes. Science 330, 502-505 (2010).

47. Roos, A. \& Boron, W. F. Intracellular pH. Physiol. Rev. 61, 296-434 (1981)

48. Jorgacevski, J. et al. Munc18-1 tuning of vesicle merger and fusion pore properties. J. Neurosci. 31, 9055-9066 (2011).

49. Schwartz, J. P. \& Wilson, D. J. Preparation and characterization of type 1 astrocytes cultured from adult rat cortex, cerebellum, and striatum. Glia 5, 75-80 (1992).

50. Miyawaki, A. et al. Fluorescent indicators for $\mathrm{Ca}^{2+}$ based on green fluorescent proteins and calmodulin. Nature 388, 882-887 (1997).

51. Nagai, T. et al. A variant of yellow fluorescent protein with fast and efficient maturation for cell-biological applications. Nat. Biotechnol. 20, 87-90 (2002).

52. Sankaranarayanan, S., De Angelis, D., Rothman, J. E. \& Ryan, T. A. The use of pHluorins for optical measurements of presynaptic activity. Biophys. J. 79, 2199-2208 (2000).

53. Balaji, J. \& Ryan, T. A. Single-vesicle imaging reveals that synaptic vesicle exocytosis and endocytosis are coupled by a single stochastic mode. Proc. Natl Acad. Sci. USA 104, 20576-20581 (2007)

\section{Acknowledgements}

We thank the financial support from EduGlia-ITN Network (EU, FP7), and grants (\# P3-310, J3-4051, J3-4146) from Slovenian Research Agency (ARRS), National Science Foundation (CBET 0943343), and National Institutes of Health (The Eunice Kennedy Shriver National Institute of Child Health and Human Development award HD078678) is acknowledged. We also thank UAB High Resolution Imaging Facility and the Carl Zeiss Reference Center for Confocal Micorscopy Ljubljana for the use of Zeiss LSM 510 and 710 confocal microscopes and the Elyra PS1 Carl Zeiss super-resolution platform. The Heflin Center Genomics Core at UAB provided sequencing for the DNA plasmids used in this work. We kindly thank Dr Matjaž Stenovec, Dr Helena Haque Chowdhury, Dr Boštjan Rituper and Dr Nina Vardjan for their help. We thank Dr Erwin Neher and Dr Bertil Hille for comments on an earlier version of the manuscript.

\section{Author contributions}

R.Z., M.K., J.J. and M.P. designed the study. P.S. performed experiments and data analysis. V.G, R.S. and V.P. contributed plasmids and experiments/analysis/associated write-ups for Supplementary Fig. 1. P.S., M.K., J.J., V.P. and R.Z. wrote the paper. All authors participated in the scientific discussions and manuscript preparation.

\section{Additional information}

Supplementary Information accompanies this paper at http://www.nature.com/ naturecommunications

Competing financial interests: The authors declare no competing financial interests.

Reprints and permission information is available online at http://npg.nature.com/ reprintsandpermissions/

How to cite this article: Singh, P. et al. Single-vesicle architecture of synaptobrevin2 in astrocytes. Nat. Commun. 5:3780 doi: 10.1038/ncomms4780 (2014). 